\title{
Novel poly(butylene succinate) nanocomposites containing strontium hydroxyapatite nanorods with enhanced osteoconductivity for tissue engineering applications
}

\author{
M. Nerantzaki ${ }^{1}$, M. Filippousi ${ }^{2}$, G. Van Tendeloo ${ }^{2}$, Z. Terzopoulou ${ }^{1}$, D. Bikiaris ${ }^{1}{ }^{*}$, O. M. Goudouri ${ }^{3}$, \\ R. Detsch ${ }^{3}$, A. Grüenewald ${ }^{3}$, A. R. Boccaccini ${ }^{3}$ \\ ${ }^{1}$ Aristotle University of Thessaloniki, GR-541 24 Thessaloniki, Macedonia, Greece \\ ${ }^{2}$ EMAT, University of Antwerp, Groenenborgerlaan 171, B-2020 Antwerp, Belgium \\ ${ }^{3}$ Institute of Biomaterials, Department of Materials Science and Engineering, University of Erlangen-Nuremberg, \\ Cauerstrasse 6, 91058 Erlangen, Germany
}

Received 31 January 2015; accepted in revised form 6 April 2015

\begin{abstract}
Three series of poly(butylene succinate) (PBSu) nanocomposites containing $0.5,1$ and $2.5 \mathrm{wt} \%$ strontium hydroxyapatite $\left[\mathrm{Sr}_{5}\left(\mathrm{PO}_{4}\right)_{3} \mathrm{OH}\right]$ nanorods (SrHAp nrds) were prepared by in situ polymerisation. The structural effects of $\mathrm{Sr}_{5}\left(\mathrm{PO}_{4}\right)_{3} \mathrm{OH}$ nanorods, for the different concentrations, inside the polymeric matrix (PBSu), were studied through high angle annular dark field scanning transmission electron microscopy (HAADF-STEM). HAADF-STEM measurements revealed that the SrHAp nanorods at low concentrations are dispersed inside the polymeric PBSu matrix while in $1 \mathrm{wt} \%$ some aggregates are formed. These aggregations affect the mechanical properties giving an enhancement for the concentration of $0.5 \mathrm{wt} \%$ SrHAp nrds in tensile strength, while a reduction is recorded for higher loadings of the nanofiller. Studies on enzymatic hydrolysis revealed that all nanocomposites present higher hydrolysis rates than neat PBSu, indicating that nanorods accelerate the hydrolysis degradation process. In vitro bioactivity tests prove that SrHAp nrds promote the formation of hydroxyapatite on the PBSu surface. All nanocomposites were tested also in relevant cell culture using osteoblast-like cells (MG-63) to demonstrate their biocompatibility showing SrHAp nanorods support cell attachment.
\end{abstract}

Keywords: nanocomposites, poly(butylene succinate), strontium nanorods, scaffolds

\section{Introduction}

Polymers are versatile materials and are used in many applications including biomedical applications because of their biocompatibility and to their modifiable properties [1,2]. Biodegradable aliphatic polyesters can be prepared to provide a wide range of degradation rates and mechanical properties [3]. Especially those belonging to the family of poly(lactic acid) (PLA) and poly(glycolic acid) (PGA), play an increasingly important role in the fledgling field of tissue engineering because they can be fashioned into porous scaffolds or carriers of cells, extracellular matrix components, and bioactive agents [4]. Poly (butylene succinate) (PBSu) is also a type of biodegradable aliphatic polyester, which can be efficiently synthesized from the starting materials of succinic acid and butan-1,4-diol. When compared with PLA and PGA, PBSu has better processability and mechanical properties similar to those of polyethylene and polypropylene [5]. Biocompatibility and osteoconductive properties of PBSu have also been shown by its ability to allow cellular adhesion and proliferation both in vitro and in vivo [6,7].

\footnotetext{
${ }^{*}$ Corresponding author, e-mail: dbic@chem.auth.gr

(C) BME-PT
} 
Moreover research has shown that all living systems are governed by molecular behaviour at nanoscale. Nanometer structural components are thus being considered as promising biomaterials [8]. Studies in the literature have shown significant increases in bone cell functions when nanophase (compared to conventional) ceramic particles are incorporated into polymer composites. Specifically, up to three times more osteoblasts adhered to PLGA when it contained nanophase particles compared to conventional titania at the same weight ratio and porosity. Moreover, significantly greater in vitro osteoblast functions leading to mineral deposition were observed on carbon fibers with nanometer size compared to conventional dimensions [8]. These results suggest that the proactive surface roughness of nanophase materials may be transferable to polymers to promote orthopedic implant efficacy.

In order to promote tissue regeneration and to modulate cellular function, researchers have also focused on the challenge of designing bone tissue engineering scaffolds that mimic the unique mechanical properties of bone. Nanophase reinforcement has the potential of improving current materials to achieve mechanical strength comparable to the native tissue. Recently, carbon nanotubes and alumoxane nanoparticles have been examined as reinforcing fillers for biodegradable polymers. The effect of a filler on the mechanical properties depends on the size, shape, and dispersion of the filler. Furthermore, the interaction between the filler and the organic matrix can also impact the level of reinforcement. Therefore, optimal performance is achieved when the small particles are uniformly dispersed throughout the polymer and interact strongly with the organic matrix.

Recently, PBSu nanocomposites containing $\mathrm{SiO}_{2}$ nanotubes and SrHAp nanorods at relatively high concentrations 5 and $20 \mathrm{wt} \%$ were prepared by melt mixing and studied as potential materials for tissue engineering applications [9]. Concerning of Sr-substituted hydroxyapatite SrHAp nrds could stimulate osteoblasts proliferation in vitro and provide an ideal environment for colonization, proliferation, and differentiation of osteoblasts to form new bone in vivo, due to its numerous phosphate groups, which could have a positive effect on cell growth. The results confirmed $\mathrm{SiO}_{2}$ nanotubes to support cell attachment and growth. However, the presence of strontium led to cytotoxicity of the nanocomposites, which might be related to critical concentration of Sr being released from the composite material.

The aim of the present study is the preparation and study of PBSu nanocomposites with optimized strontium hydroxyapatite nanorods' concentrations for tissue engineering applications. As an alternative development method in situ polymerization will be used, in order to achieve a more uniform dispersion of the nanofillers in the polymer matrix and thus improve the physical properties. Compared with the method of melt mixing, in situ polymerization gives more satisfactory dispersion of nanoparticles and stronger interactions between the reinforcements and the polymeric phase. Therefore, nanocomposites fabricated by in situ processing tend to display better mechanical properties and a lower percolation threshold than those composites made by ex situ methods.

\section{Experimental \\ 2.1. Materials}

Succinic acid (CAS Number: 110-15-6) (purum 99+\%), 1,4-butanediol (110-63-4) (purity >99\%) and titanium (IV) butoxide (TBT) (5593-70-4) $(>97 \%)$ were obtained from Sigma-Aldrich Chemical Co (Chemie GmbH, Germany) and used as received. Strontium nitrate $\left[\mathrm{Sr}\left(\mathrm{NO}_{3}\right)_{2}\right]$ (10042-76-9) $(\geq 99 \%)$, ammonium phosphate dibasic $\left[\left(\mathrm{NH}_{4}\right)_{2} \mathrm{HPO}_{4}\right]$ (7783-28-0) ( $\geq 98 \%$ ) and trisodium citrate dehydrate (labelled as $\mathrm{Cit}^{3-}$ ) (6132-04-3) ( $\left.\geq 99 \%\right)$ were supplied from Sigma Aldrich Chemical Co (Chemie $\mathrm{GmbH}$, Germany). Cetyltrimethyl-ammonium bromide (57-09-0) (labelled as CTAB) ( $\geq 99 \%$ ) was purchased from Panreac Quimicha (Panreac Quimica S.L.U., Spain). All other materials and solvents used in the analytical methods were of analytical grade.

\subsection{Synthesis of strontium hydroxyapatite nanorods}

Strontium hydroxyapatite nanorods were synthesized via the hydrothermal method that Zhang et al. [10] previously described. In a typical procedure, $3 \mathrm{mmol}$ of $\operatorname{Sr}\left(\mathrm{NO}_{3}\right)_{2}, 0.5 \mathrm{~g}$ of CTAB, and $10 \mathrm{~mL}$ of ammonia solution $\left(\mathrm{NH}_{3} \cdot \mathrm{H}_{2} \mathrm{O}\right)$ (used for adjusting the $\mathrm{pH}$ value as alkaline solution) were dissolved in $30 \mathrm{~mL}$ of deionized water to form solution 1 . Then, $6 \mathrm{mmol}$ of trisodium citrate (labeled as $\mathrm{Cit}^{3-}$, the molar ratio of $\mathrm{Cit}^{3-} / \mathrm{Sr}^{2-}$ is $2: 1$ ) and $2 \mathrm{mmol}$ of $\left(\mathrm{NH}_{4}\right)_{2} \mathrm{HPO}_{4}$ were added into $20 \mathrm{~mL} \mathrm{H}_{2} \mathrm{O}$ to form solution 2 . After vigorously stirring for $30 \mathrm{~min}$, solu- 
tion 2 was introduced into solution 1 (dropwise). After additional agitation for $20 \mathrm{~min}$, the as-obtained mixing solution was transferred into a Teflon bottle $(80 \mathrm{~mL})$ held in a stainless steel autoclave, sealed, and maintained at $180^{\circ} \mathrm{C}$ for $24 \mathrm{~h}$. As the autoclave cooled to room temperature naturally, the precipitate was separated by centrifugation, washed with deionized water and ethanol in sequence. Then, the obtained product was redispersed in $150 \mathrm{~mL}$ of acetone and refluxed at $80^{\circ} \mathrm{C}$ for $48 \mathrm{~h}$ to remove the residual template CTAB. Finally, the precipitate was separated by centrifugation again and dried in vacuum at $70^{\circ} \mathrm{C}$ for $24 \mathrm{~h}$ to obtain the final sample [10].

\subsection{In situ prepared nanocomposites}

Nanocomposites of PBSu and SrHAp nanorods were prepared in situ by the two-stage melt polycondensation method using succinic acid (SA) and 1,4butanediol (BD) in a glass batch reactor at a molar ratio SA:BD of 1:1.15. Appropriate amount of SrHAp nrds was first dispersed in 1,4-butanediol by ultrasonic vibration ( $50 \mathrm{~W}$, Hielscher UP50H) and intense stirring with a magnetic stirrer (300 rpm) for $10 \mathrm{~min}$ prior to polymerization. The polymerization mixture, after being placed in a $250 \mathrm{~cm}^{3}$ round bottom flask, was de-aired and purged with dry nitrogen three times. Thereupon, the mixture was heated under a nitrogen atmosphere for $3.5 \mathrm{~h}$ at $200^{\circ} \mathrm{C}$ under constant stirring (350 rpm), with water removed as the reaction by-product of esterification. Afterwards, for the second reaction stage of polycondensation, $0.3 \mathrm{wt} \%$ of TPP as heat stabilizer and $1.0 \cdot 10^{-3} \mathrm{~mol}$ per mole of succinic acid of TBT as polycondensation catalyst were added. The reaction was continued under increased mechanical stirring $(720 \mathrm{rpm})$ and high vacuum $(\sim 5.0 \mathrm{~Pa})$, which was applied slowly over a period of time of about $30 \mathrm{~min}$, to avoid excessive foaming and to minimize oligomer sublimation, at 220 and $240^{\circ} \mathrm{C}$ for $1 \mathrm{~h}$ intervals, respectively. Polymerization was stopped by fast cooling to room temperature. After the polycondensation reaction was completed, the polyesters were easily removed from the flask. According to this procedure neat PBSu and nanocomposites containing 0.5, 1 and $2.5 \mathrm{wt} \%$ SrHAp nrds have been prepared.

\subsection{Nanocomposites characterization}

\subsubsection{Gel permeation chromatography (GPC)}

GPC analysis was performed using a Waters 150C GPC (Waters Corp., Milford, MA) equipped with a differential refractometer as detector and three ultrastyragel $(103,104,105 \mathrm{E})$ columns in series. $\mathrm{CHCl}_{3}$ was used as the eluent $(1 \mathrm{~mL} / \mathrm{min})$ and the measurements were performed at $35^{\circ} \mathrm{C}$. Calibration was performed using polystyrene standards with a narrow molecular weight distribution.

\subsubsection{Fourier Transformed-Infrared Spectroscopy (FTIR)}

FTIR spectra were obtained using a Perkin-Elmer FTIR (Perkin-Elmer Inc. USA) spectrometer, model Spectrum One. The materials were in the form of thin films with a thickness of approximately $30 \mu \mathrm{m}$. The IR spectra of these films were obtained in absorbance mode and in the spectral region of 400$4000 \mathrm{~cm}^{-1}$ using a resolution of $4 \mathrm{~cm}^{-1}$ and $64 \mathrm{co-}$ added scans.

\subsubsection{X-ray photoelectron spectroscopy (XPS)}

The element composition and interfacial interactions were studied using X-ray photoelectron spectroscopy (XPS). In the XPS analysis (AXIS Ultra 'DLD' X-ray photoelectron spectrometer) (Shimadzu Corp., Japan), a monochromatic Al Ka X-ray was used at $14 \mathrm{kV}$. A binding energy of $284.8 \mathrm{eV}$ for the $\mathrm{C} 1 \mathrm{~s}$ of aliphatic carbons was taken as the reference energy. The XPS curve fitting of $\mathrm{C} 1 \mathrm{~s}, \mathrm{O} 1 \mathrm{~s}$ and $\mathrm{Sr} 3 \mathrm{~d}$ were accomplished using Kratos' Vision 2 Processing software.

\subsubsection{Scanning electron microscopy (SEM)}

The morphology of the prepared nanocomposites was examined using a scanning electron microscopy system (SEM) type JEOL (JMS-840) (Jeol Ltd., Tokyo) equipped with an energy-dispersive X-ray (EDX) Oxford ISIS 300 micro-analytical system (Oxford Instruments UK). The studied surfaces were coated with carbon black in order to obtain good conductivity for the electron beam. Operating conditions were: accelerating voltage $20 \mathrm{kV}$, probe current $45 \mathrm{nA}$ and counting time $60 \mathrm{~s}$.

\subsubsection{High angle annular dark field scanning transmission electron microscopy}

Samples suitable for HAADF-STEM measurements were sectioned using a Leica UC7 ultramicrotome equipped with a FC7 cryo chamber and the sections had a thickness of 100-120 nm. The sections were placed on Quantifoil C coated Cu grids. Samples were cut at $-80^{\circ} \mathrm{C}$. HAADF-STEM images were 
acquired using a FEI Tecnai G2 electron microscope operated at $200 \mathrm{kV}$.

\subsubsection{X-ray Diffractometry (WAXD)}

XRD studies of nanocomposites, in the form of thin films, were performed over the range $2 \mathrm{~h}$ from 5 to $80^{\circ}$, at steps of $0.05^{\circ}$ and counting time of $5 \mathrm{~s}$, using a MiniFlex II XRD system from (RIGAKU Co., Ltd., Japan). The degree of crystallinity was measured using instrument's software.

\subsubsection{Mechanical properties}

Measurements of mechanical properties of the prepared nanocomposites in tension were performed on an Instron 3344 dynamometer (Instron, USA) in accordance with ASTM D638, using a crosshead speed of $5 \mathrm{~mm} / \mathrm{min}$. Relative thin sheets of about $350 \pm 25 \mu \mathrm{m}$ were prepared using an Otto Weber, Type PW 30 hydraulic press connected with an Omron E5AX Temperature Controller, at a temperature of $130 \pm 5^{\circ} \mathrm{C}$. The moulds were rapidly cooled by immersing them in water at $20^{\circ} \mathrm{C}$ In order to measure the mechanical properties from these sheets, dumb-bell-shaped tensile test specimens (central portions $5 \times 0.5 \mathrm{~mm}$ thick, $22 \mathrm{~mm}$ gauge length) were cut in a Wallace cutting press and conditioned at $25^{\circ} \mathrm{C}$ and $55-60 \%$ relative humidity for $48 \mathrm{~h}$. The values of Young's modulus, yield stress, elongation at break and tensile strength at the break point were determined. At least five specimens were tested for each sample and the average values are reported.

\subsubsection{Dynamic mechanical analysis (DMA)}

The dynamic mechanical properties of the nanocomposites were measured with a Perkin-Elmer Diamond DMA (PerkinElmer, Inc. USA). The bending (dual cantilever) method was used with a frequency of $1 \mathrm{~Hz}$, a strain level of $0.04 \%$, in the temperature range of -70.0 to $80.0^{\circ} \mathrm{C}$. The heating rate was $3^{\circ} \mathrm{C} / \mathrm{min}^{-1}$. Testing was performed using rectangular bars measuring approximately $30 \times 10 \times 3.0 \mathrm{~mm}^{3}$. These were prepared with a hydraulic press, as described above, and the exact dimensions of each sample were measured before the scan.

\subsubsection{Contact angle measurements}

Contact angle measurements, before and after sterilization, were performed using a DSA30 analyzer, (KRUSS GmbH, Germany). The samples were in the form of thin films of $500 \pm 50 \mu \mathrm{m}$ thickness and
$0.5 \mathrm{~cm}$ diameter. The drop of water used had a diameter of $3 \mu \mathrm{m}$.

\subsubsection{Enzymatic hydrolysis}

Samples in the form of films $\left(10 \times 20 \times 0.4 \mathrm{~mm}^{3}\right)$ prepared using an Otto Weber Type PW 30 hydraulic press at $130^{\circ} \mathrm{C}$, were placed in test tubes, wherein $5 \mathrm{~mL}$ of phosphate buffer solution $(0.2 \mathrm{M}, \mathrm{pH} 7.0)$ was added, containing Rhizopus delemar $\geq 0.4 \mathrm{U} / \mathrm{mg}$ and Pseudomonas Cepacia $\geq 30 \mathrm{U} / \mathrm{mg}$ lipase. The concentrations of the lipases were 0.09 and $0.01 \mathrm{mg} / \mathrm{mL}$, respectively, because $0.1 \mathrm{mg} / \mathrm{mL}$ total enzyme concentration in PBS is sufficient to cause notable mass loss over a period of 40 days of enzymatic treatment at $37^{\circ} \mathrm{C}$. The loosely capped test tubes were kept at $37.0 \pm 1.0^{\circ} \mathrm{C}$ in an oven for several days while the media were replaced every 3 days. After a predetermined time the films were removed from the lipase solution, washed thoroughly with distilled water and ethanol and then dried at room temperature under vacuum, until constant weight. Every measurement was repeated three times. As a control, the same experiment was carried out under the same conditions without any lipase being added. The degree of biodegradation was estimated from the mass loss. The morphology of the prepared films during enzymatic hydrolysis was examined through scanning electron micrographs (SEM).

\subsubsection{Formation of bone-like apatite}

After ultrasonic cleaning in acetone and rinsing with distilled water, all the samples were soaked in a simulated body fluid (SBF) for 14 and 28 days in order to assess their bioactivity. The ionic concentrations in the SBF solution are nearly equal to those in human body blood plasma, and the solution was buffered at $\mathrm{pH} 7.4$ with trimethanol aminomethaneHCL. After the immersion test, each specimen was gently washed with distilled water, gradually dried with ethanol. Finally, the specimens were observed by scanning electron microscopy.

\subsubsection{Cell culture studies}

The in vitro biocompatibility of all materials was evaluated in contact with MG63 osteoblast-like cells after $48 \mathrm{~h}$ of incubation. Six samples from each category were disinfected by immersion in $70 \mathrm{vol} \%$ ethanol solution for $1 \mathrm{~h}$. After disinfection the samples were washed with phosphate-buffered saline (PBS, Gibco ${ }^{\circledR}$ ) and were placed into a single well of 
a 24-well plate (Corning, Cambridge, MA). $1.5 \mathrm{~mL}$ of Dulbecco's Modified Eagle's medium (DMEM, Gibco, Germany) supplemented with 10 vol $\%$ fetal bovine serum (FBS, Sigma-Aldrich, Germany) and 1 vol\% penicillin and streptomycin (Gibco, Germany) containing $10^{5}$ cells was added to each well. The same amount of culture medium with cells without specimens was used as positive control. All samples and controls were incubated at $37^{\circ} \mathrm{C}$ in a humidified atmosphere $\left(5 \% \mathrm{CO}_{2}\right.$ in $95 \%$ air, SteriCycle, Thermo Fisher Scientific) for 48 hours. Afterwards, the samples were washed with PBS and $1 \mathrm{~mL}$ of AlamarBlue solution was added in the well plate. The well plate was incubated at $37^{\circ} \mathrm{C}(95 \%$ humidity, $5 \% \mathrm{CO}_{2}$ ) for 4 hours. The AlamarBlue solution was prepared by mixing $10 \mathrm{vol} \%$ of Alamar Blue reagent (Invitrogen, Germany) with DMEM supplemented with FCS and antibiotics as previously described. After 4 hours of incubation, the optical density of the solutions was measured at 570 and $600 \mathrm{~nm}$ with a plate reader (Anthos, Germany). Cell morphologies were obtained by SEM microscopy (Quanta 200, FEI, The Netherlands). All samples were washed with PBS, fixed with a solution containing 3 vol $\%$ glutaraldehyde (Sigma, Germany) and 3 vol \% paraformaldehyde (Sigma, Germany) in $0.2 \mathrm{M}$ sodium cacodylate buffer ( $\mathrm{pH} 7.4$ ) and finally rinsed three times with PBS. Consequently, all samples were dehydrated in a graded ethanol series (30, 50, 70, 80, 90, 95, and 99.8 vol\%). Samples were maintained in $99.8 \mathrm{vol} \%$ ethanol and critical-point dried (EM CPD300, Leica, Germany) before imaging with SEM.

\section{Results and discussion}

\subsection{Synthesis and characterization of in situ prepared PBSu/SrHAp nrds nanocomposites}

PBSu nanocomposites were synthesized by twostep reactions of esterification and polycondensation. According to this procedure polyesters with high molecular weight can be prepared and the $M_{\mathrm{n}}$ and $M_{\mathrm{w}}$ values of neat PBSu are calculated at 40850 and $92220 \mathrm{~g} / \mathrm{mol}$, respectively, which is quite satisfactory for aliphatic polyesters [11]. The presence of the nanorods in the polymerization mixture, affected the reaction, leading to variations of the final molecular weight of the synthesized polymers. As shown in Table 1, the average molecular weight of the nanocomposites varies. Initially the molecular weight was
Table 1. Molecular weight of prepared nanocomposites

\begin{tabular}{|l|c|c|c|}
\hline \multicolumn{1}{|c|}{ Sample } & $\begin{array}{c}\mathbf{M}_{\mathbf{n}} \\
{[\mathbf{g} / \mathbf{m o l}]}\end{array}$ & $\begin{array}{c}\mathbf{M}_{\mathbf{w}} \\
{[\mathbf{g} / \mathbf{m o l}]}\end{array}$ & $\mathbf{M}_{\mathbf{w}} / \mathbf{M}_{\mathbf{n}}$ \\
\hline PBSu & 40850 & 92220 & 2.3 \\
\hline PBSu/SrHAp nrds 0.5 wt\% & 51100 & 122950 & 2.4 \\
\hline PBSu/SrHAp nrds 1.0 wt\% & 37790 & 83380 & 2.2 \\
\hline PBSu/SrHAp nrds 2.5 wt\% & 21550 & 41010 & 1.9 \\
\hline
\end{tabular}

increased for a SrHAp nanorod concentration of $0.5 \mathrm{wt} \%$, followed by a gradual decrease at higher concentrations. This is due to the interactions that probably are taking place between nanorods and PBSu macromolecules, since SrHAp nanorods have a lot of hydroxyl groups that could react with the $-\mathrm{COOH}$ or $-\mathrm{OH}$ end groups of PBSu macromolecules.

Due to these interactions at a low concentration the nanoparticles could act as chain extenders, increasing the molecular weight of the polyester, and thus macromolecules with higher molecular weight than pure polyester were produced, as in the nanocomposite containing $0.5 \mathrm{wt} \% \mathrm{SrHAp}$ nrds. However, at higher concentrations, due to extended reactions, branched and/or cross-linked macromolecules could be formed, since SrHAp nrds, due to the surface hydroxyl groups, can act as a multifunctional agent, leading to a partial reduction of molecular weight [12], as in nanocomposites containing 1.0 and $2.5 \mathrm{wt} \%$ SrHAp nanorods. Branched polymers generally have lower intrinsic viscosities than the corresponding linear ones having the same molecular weight. This happens due to the hydrodynamic dimensions of branched macromolecules, which is what the methods used for the determination of the molecular weight actually measure, are smaller in solution than linear ones. Similar reactions were mentioned in PBSu/silica nanocomposites and it was revealed that silanol groups $(\mathrm{Si}-\mathrm{OH})$ in $\mathrm{SiO}_{2}$ nanoparticles could react with the hydroxyl end groups of the polyesters. Such a reaction was verified using results from solid-state ${ }^{29} \mathrm{Si} \mathrm{NMR}$ and FTIR spectra of PBSu/silica nanocomposites, indicating covalent bonding between $\mathrm{SiO}_{2}$ particles and the PBSu macromolecular chain [13-15].

In the present study FTIR and XPS were used to examine the possibility of such reactions to take place between $\mathrm{Sr}-\mathrm{OH}$ groups of SrHAp nanorods and PBSu. As shown in Figure 1 for the neat PBSu sample, the characteristic ester absorption peak for the stretching vibration of the $-\mathrm{C}=\mathrm{O}$ bond appears at $1717 \mathrm{~cm}^{-1}$. The peak at $2960 \mathrm{~cm}^{-1}$ is attributed to 
the stretching vibration of $\mathrm{C}-\mathrm{H}$ of the $-\mathrm{CH} 2$ - group while the wide peak at $3100-3700 \mathrm{~cm}^{-1}$ corresponds to hydroxyl groups of the polyester. For the PBSu nanocomposites, the infrared spectra retained most of the band of PBSu, although some of them changed in intensity. Thus, it seems that no interactions between inorganic nanoparticles and PBSu matrix took place and covalently bonds between PBSu and

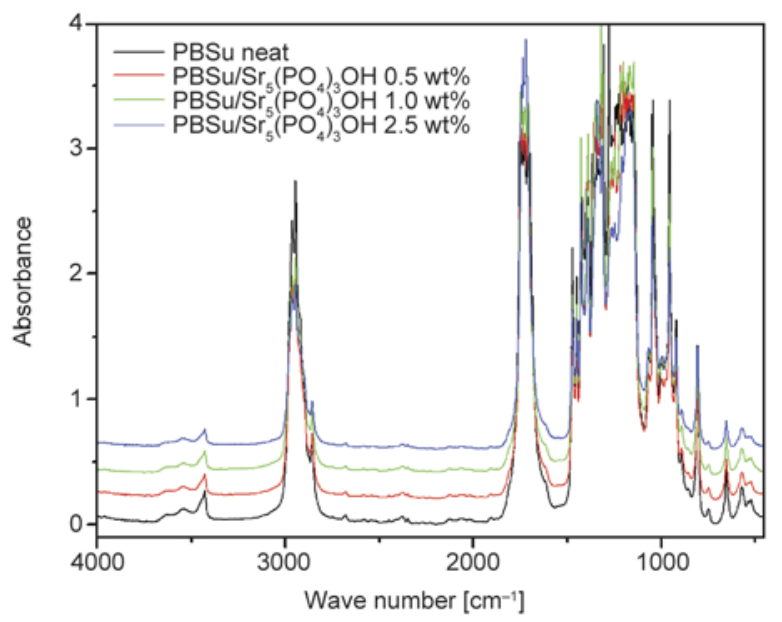

Figure 1. FTIR spectra of prepared nanocomposites

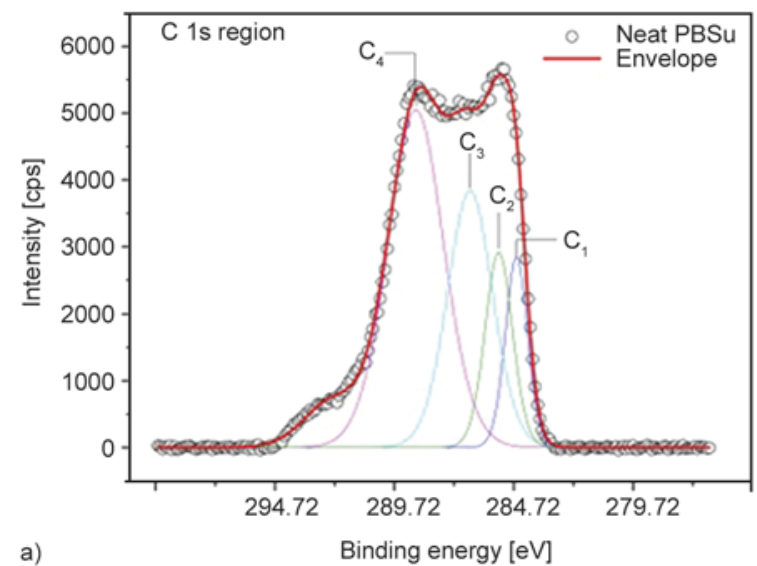

SrHAp nanorods have not been formed. However, there is also the possibility due to the low amount of added SrHAp nanorods, these interactions could not be detected with FTIR, due to its low sensitivity.

In order to investigate the possible covalent bonding between SrHAp nanorods and the PBSu polymer backbone chain, XPS analysis was performed. Neat PBSu has characteristic peaks at the regions $\mathrm{C} 1 \mathrm{~s}$ and $\mathrm{O} 1 \mathrm{~s}$ with binding energies 283-292 and $529-536 \mathrm{eV}$, respectively (Figure 2). Both peaks can be further analyzed to different binding energies corresponding to different carbonyl components like $\mathrm{C}_{1}=\mathrm{CC}$ and $\mathrm{CH}(284.52 \mathrm{eV}), \mathrm{C}_{2}=\mathrm{bC}$ to ester $(285.32 \mathrm{eV}), \mathrm{C}_{3}=\mathrm{C}-\mathrm{O}(286.52 \mathrm{eV}), \mathrm{C}_{4}=$ ester $(\mathrm{C}=\mathrm{O})-\mathrm{O}(288.72 \mathrm{eV})$ (Figure 2$)$ and oxygen like $\mathrm{O}_{1}=\operatorname{ester}(\mathrm{C}=\mathrm{O})-\mathrm{O}(532.01 \mathrm{eV}), \mathrm{O}_{2}=$ carbonyl $(\mathrm{C}=\mathrm{O})(533.38 \mathrm{eV})$ and $\mathrm{O}_{3}$ carboxylic $(\mathrm{O}=\mathrm{C})-\mathrm{OH}$ $(535,42 \mathrm{eV})$ (Figure 3) [16]. XPS study was performed for all the nanocomposites, but for the samples with PBSu/SrHAp nrds $0.5 \mathrm{wt} \%$ and $\mathrm{PBSu} /$ SrHAp nrds $1.0 \mathrm{wt} \%$ the presence of the nanofiller was not confirmed. XPS analysis is a surface-sensi-

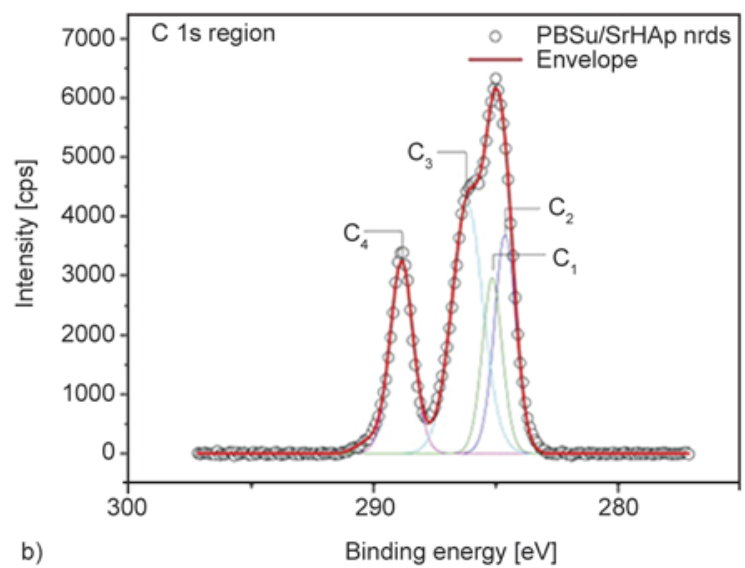

Figure 2. XPS spectra of a) PBSu and b) PBSu/SrHAp 2.5 wt $\%$ nrds in $\mathrm{C} 1 \mathrm{~s}$ region
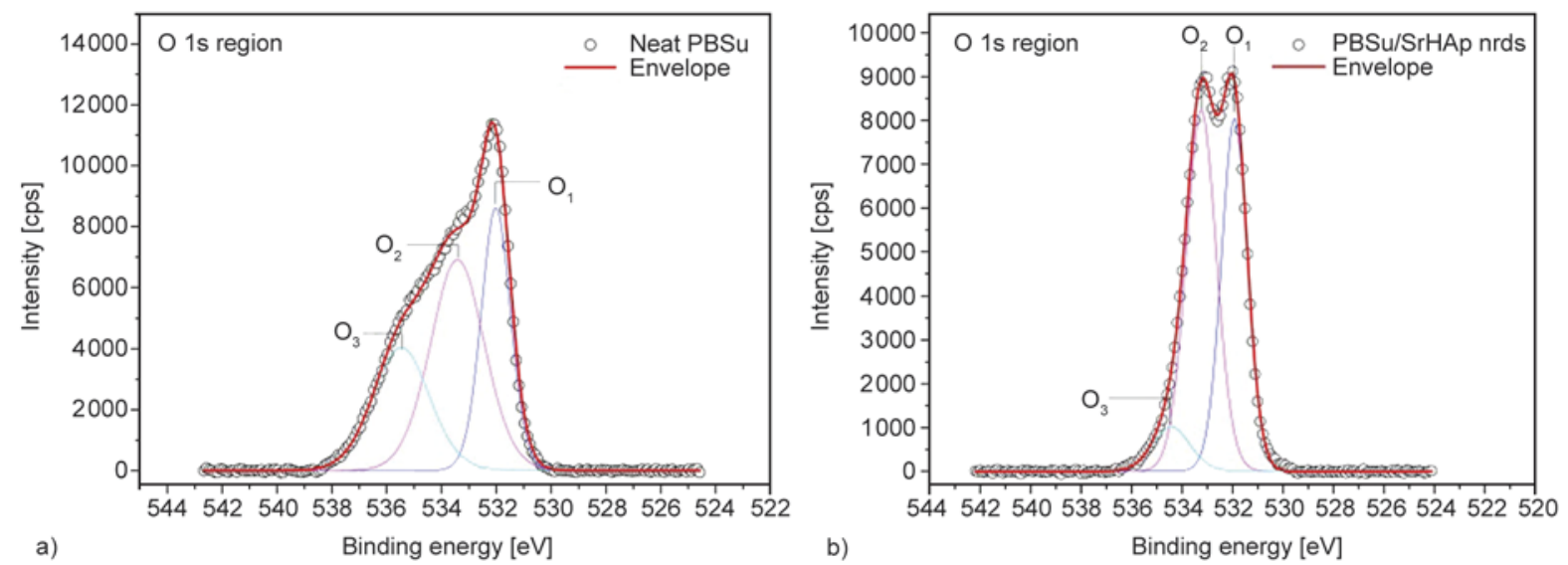

Figure 3. XPS spectra of a) PBSu and b) PBSu/SrHAp nrds $2.5 \mathrm{wt} \%$ in $\mathrm{O} 1 \mathrm{~s}$ region 
Table 2. Characteristic binding energies of carbonyl and oxygen components in $\mathrm{PBSu}$ and $\mathrm{PBSu} / \mathrm{SrHAp}$ nrds $2.5 \mathrm{wt} \%$ nanocomposite

\begin{tabular}{|c|c|c|}
\hline \multirow{2}{*}{ Component } & \multicolumn{2}{|c|}{ Peak position [eV] } \\
\cline { 2 - 3 } & NeatPBSu & PBSu/SrHAp nrds \\
\hline $\mathrm{C}_{1}$ & 284.52 & 284.6 \\
\hline $\mathrm{C}_{2}$ & 285.32 & 285.09 \\
\hline $\mathrm{C}_{3}$ & 286.52 & 286.17 \\
\hline $\mathrm{C}_{4}$ & 288.72 & 288.76 \\
\hline $\mathrm{O}_{1}$ & 532.01 & 531.92 \\
\hline $\mathrm{O}_{2}$ & 533.38 & 533.34 \\
\hline $\mathrm{O}_{3}$ & 535.42 & 534.35 \\
\hline
\end{tabular}

where: $\mathrm{C}_{1}=\mathrm{CC}$ and $\mathrm{CH}, \mathrm{C}_{2}=\mathrm{bC}$ to ester, $\mathrm{C}_{3}=\mathrm{C}-\mathrm{O}$,

$\mathrm{C}_{4}=\operatorname{ester}(\mathrm{C}=\mathrm{O})-\mathrm{O}$,

$\mathrm{O}_{1}=\operatorname{ester}(\mathrm{C}=\mathrm{O})-\mathrm{O}, \mathrm{O}_{2}=\operatorname{carbonyl}(\mathrm{C}=\mathrm{O})$ and

$\mathrm{O}_{3}=$ carboxylic $(\mathrm{O}=\mathrm{C})-\mathrm{OH}$

tive quantitative spectroscopic technique and for this reason penetration depth is low. For the nanocomposites with SrHAp nrds content less than $2.5 \mathrm{wt} \%$ it is probable that the nanofiller was embedded in the polymeric matrix during bulk polymerization and thus was not possible to be detected in order to investigate chemical bonds.

The binding energies of the sample $\mathrm{PBSu} / \mathrm{SrHAp}$ nrds $2.5 \mathrm{wt} \%$ are presented in Table 2. Comparing the carbonyl group binding energies between neat $\mathrm{PBSu}$ and its nanocomposite with $2.5 \mathrm{wt} \%$ SrHAp nrds it can be seen that these are almost identical. However, same small differences are recorded in the oxygen region and mainly of the $\mathrm{O}_{1}$ ester and $\mathrm{O}_{3}$ carboxylic group (Figure 3 ). These values are slightly to lower binding energies indicating the existence of some interactions between PBSu and these nanoparticles [17].

After the peak analysis in the Sr3d region of the XPS spectrum at (130.5-137.6 eV) for the PBSu/SrHAp nrds $2.5 \mathrm{wt} \%$ nanocomposite, it is clear that new peaks are formed, indicating the formation of new covalent bonds between SrHAp nrds nanoparticles and PBSu (Figure 4). Probably, a new chemical bond $(\mathrm{Sr}-\mathrm{O}-\mathrm{C})$ is formed by the reaction between $\mathrm{Sr}-\mathrm{OH}$ and the hydroxyl end groups of PBSu macromolecular chains. The binding energy of this new peak is $137.6 \mathrm{eV}$, while the peaks at 132.86 and $134.52 \mathrm{eV}$ more likely correspond to $\mathrm{Sr}-\mathrm{O}$ bonds of SrHAp nrds $[10,18,19]$.

Regarding WAXD analysis, many interesting results could be concluded studying XRD pattern of PBSu and its nanocomposites. However, the authors focus firstly on the confirmation of PBSu synthesis and secondly on the effect of nanofiller on the degree of

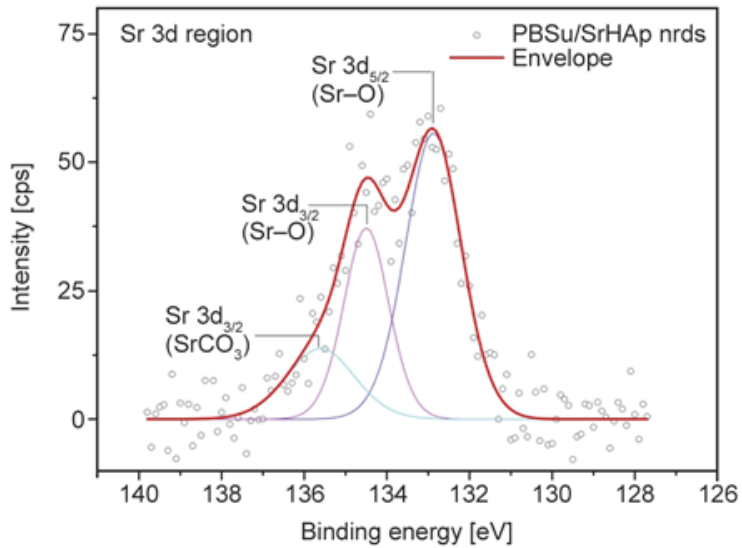

Figure 4. XPS spectra of $\mathrm{PBSu} / \mathrm{Sr}_{5}\left(\mathrm{PO}_{4}\right)_{3} 2.5 \mathrm{wt} \%$ nanocomposite in $\mathrm{Sr} 3 \mathrm{~d}$ region

crystallinity. The crystalline structure of the nanocomposites was studied using XRD. The crystal unit cell of PBSu is monoclinic and diffraction peaks from [020] and [110] are observed at 20 19.8 and $22.6^{\circ}$ respectively $[9,11,20]$. Although SrHAp nanorods are crystalline materials none of their peaks can be recognized, even these with high intensity at 20.4, 26.6 and $31^{\circ}$, because they are overlapped by denser PBSu peaks (Figure 5). In general, as no shifting of PBSu peaks is observed, we deduce that the unit cells of the polyester and nanofillers remain the same. However, the addition of SrHAp nanorods has a small effect on the PBSu degree of crystallinity, which in neat PBSu is about $41.2 \%$. This was slightly increased in nanocomposites containing $0.5,1$ and $2.4 \mathrm{wt} \%$ SrHAp nanorods to $42.5,43.1$ and $43.3 \%$, respectively. This is because SrHAp nanorods may act as nucleating agents promoting the heterogeneous nucleation of PBSu.

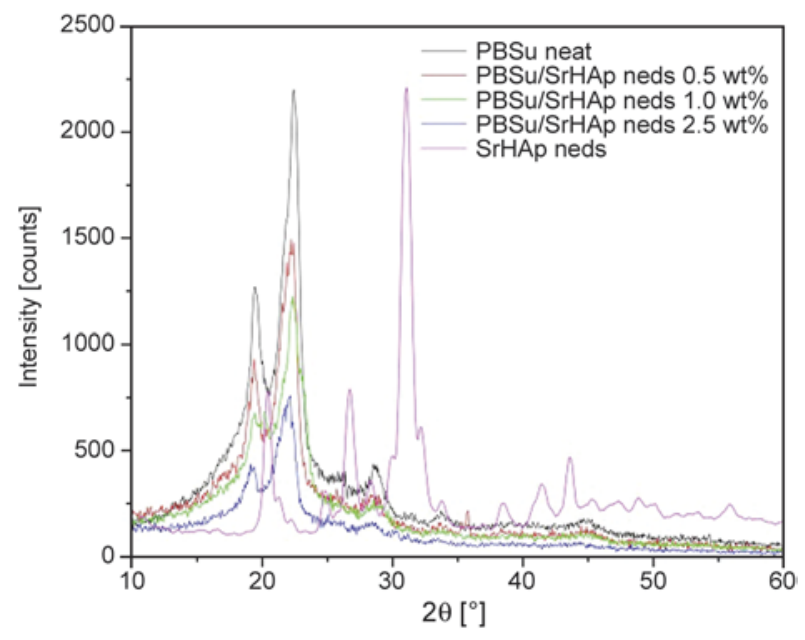

Figure 5. XRD patterns of neat PBSu, neat SrHAp nanorods and their nanocomposites containing different content of SrHAp nanorods 


\subsection{Morphological, mechanical and dynamic mechanical analysis of PBSu/SrHAp nrd nanocomposites.}

Considering that low adhesion between the used nanoadditives and the PBSu matrix could affect their dispersion, we analysed their morphological features. In Figures 6 and 7, HAADF-STEM images of the samples SrHAp nanorods 0.5 and $1.0 \mathrm{wt} \%$ respectively, inside the polymeric matrix in low and high magnification, are presented. From these images the particles seem to aggregate, even though we can observe a better dispersion for the sample consisting of $0.5 \mathrm{wt} \%$ the nanorods compared to that with the $1.0 \mathrm{wt} \%$ concentration,. It seems that the dispersion of nanorods in 1,4-butanediol by ultrasonic vibration, before their in situ addition in the PBSu matrix, contributed to the high level of dispersion $(\leq 0.5 \mu \mathrm{m})$ and distribution in the polymer matrix.

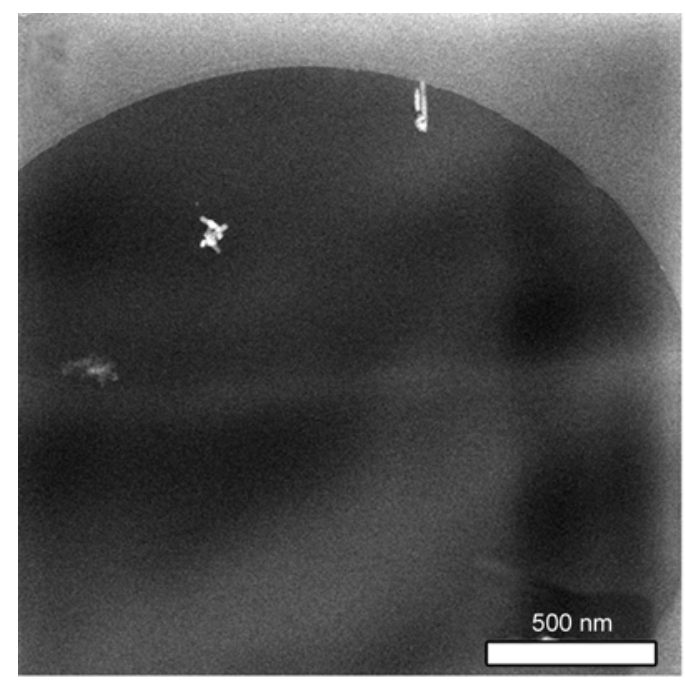

a)

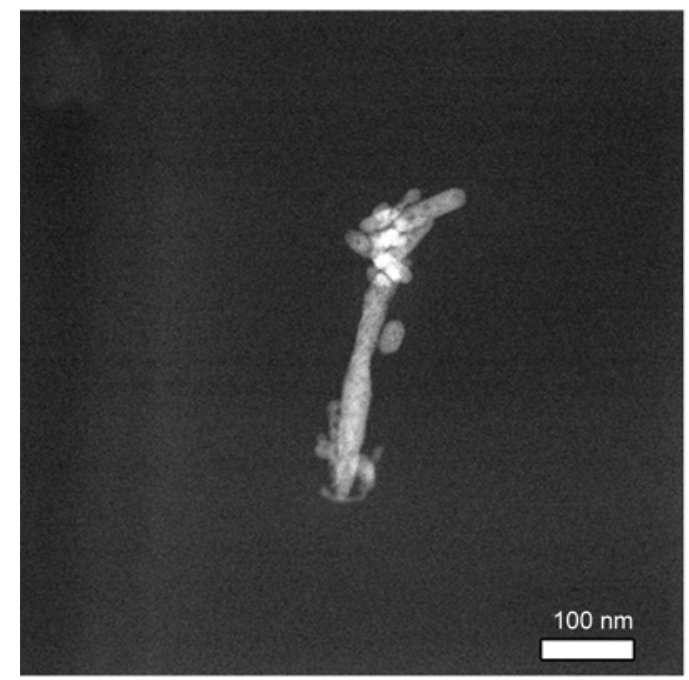

c)
This was the main problem in our previous study in which PBSu/SrHAp nrds nanocomposites have been prepared by melt mixing using 5 and $20 \mathrm{wt} \%$ SrHAp nrds [9].

It is well known that the filler dispersion inside the polymer matrix affects the mechanical properties of the materials. From the stress-strain curves of neat $\mathrm{PBSu}$ and its nanocomposites it is found that all materials are very brittle since they are breaking before yielding. This is maybe due to the low molecular weight of all materials. From these curves the tensile strength at break, elongation at break and Young's modulus were calculated and presented in Table 3.

Despite that $\mathrm{PBSu}$ is a rigid material with high Young's modulus $408 \mathrm{MPa}$, it is still brittle, as it does not withstand much elongation before breaking (elongation at break $2 \%$ ). Indeed the low flex-

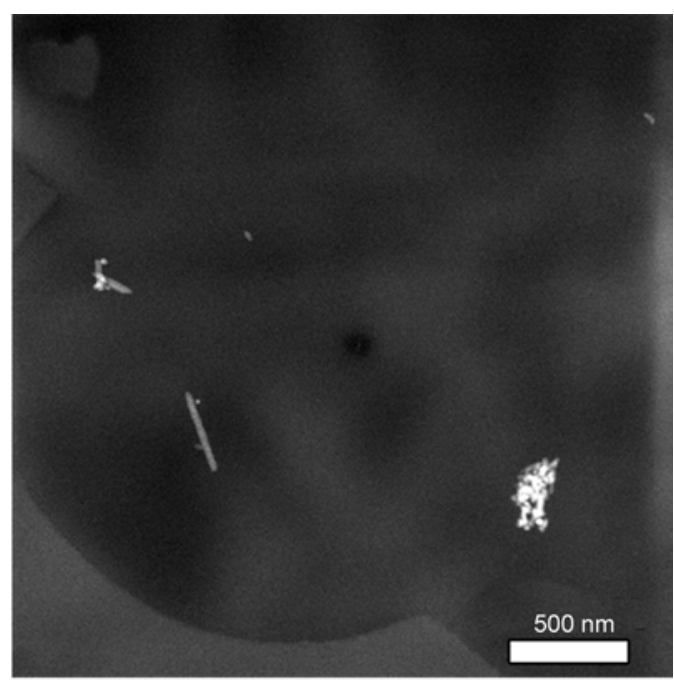

b)

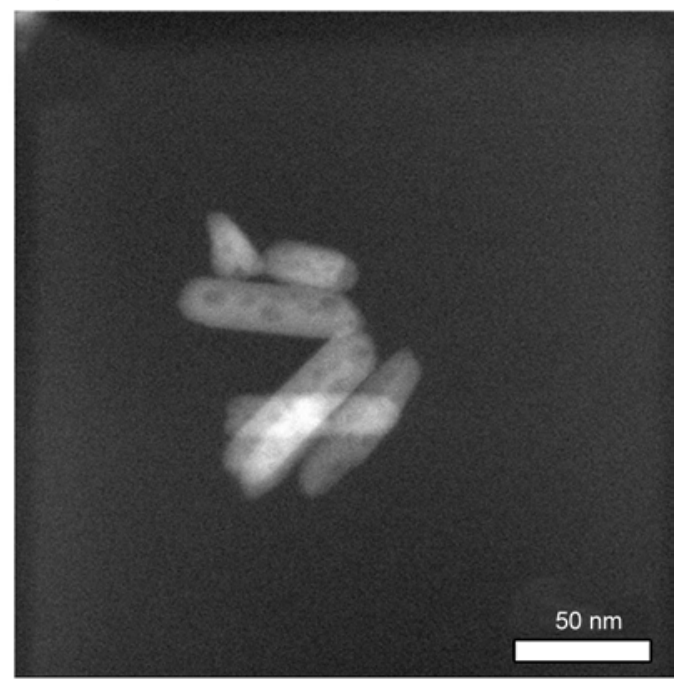

d)

Figure 6. (a)-(d) Low and high magnification HAADF-STEM images of the sample PBSu-SrHAp $0.5 \mathrm{wt} \%$ showing the dispersion of the SrHAp nanorods (brighter features) inside the polymeric matrix 


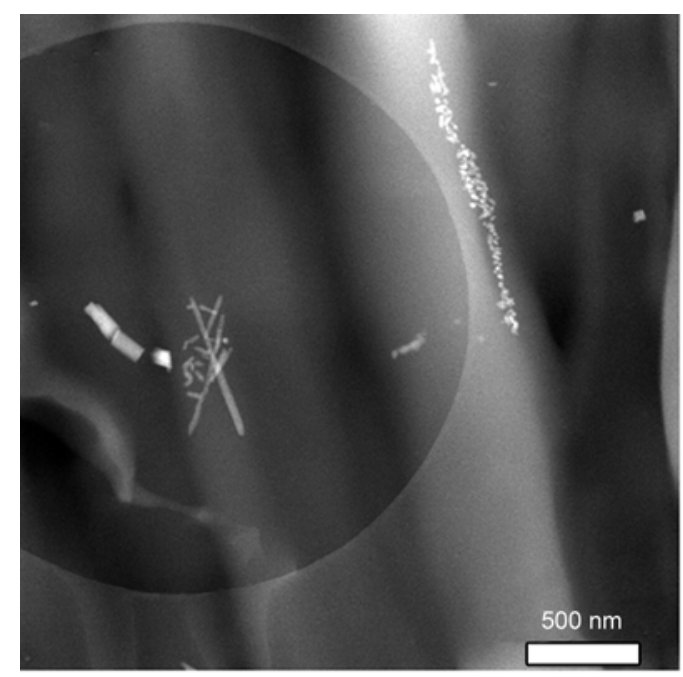

a)

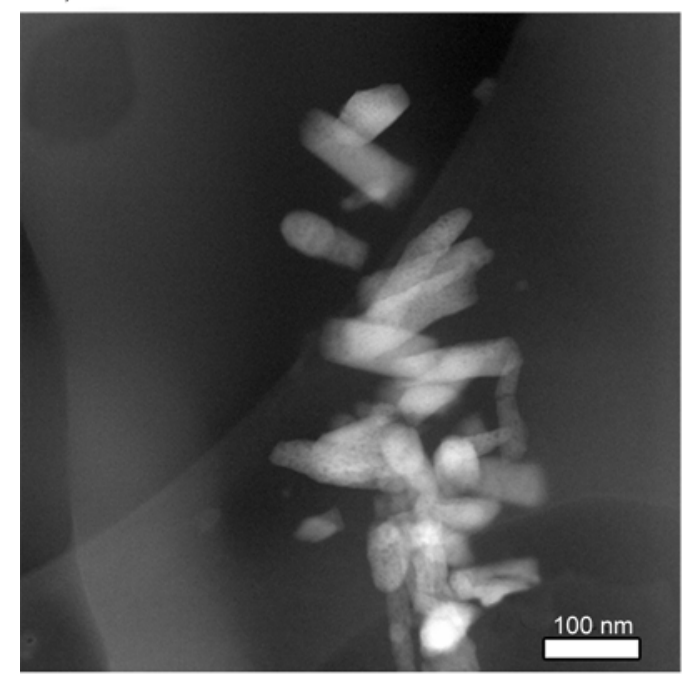

c)

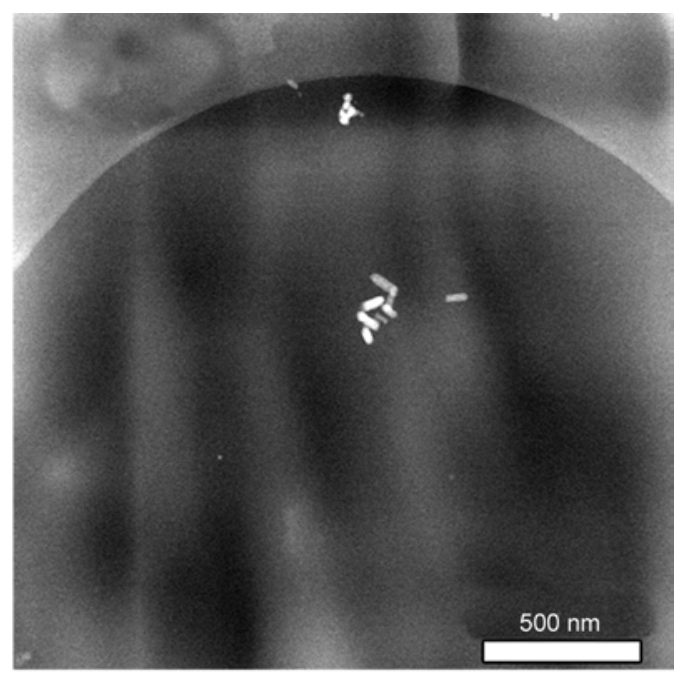

b)

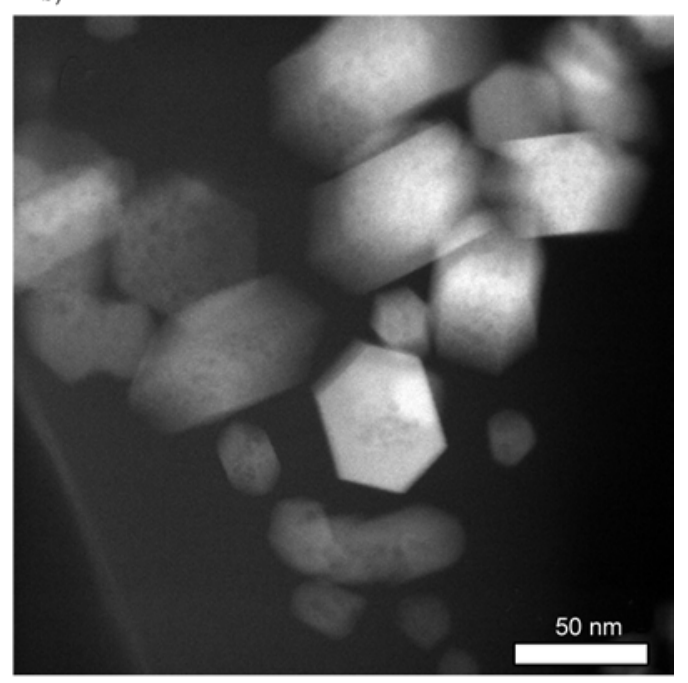

d)

Figure 7. (a)-(d) Low and high magnification HAADF-STEM images of the sample PBSu-SrHAp 1.0 wt $\%$ showing the dispersion of the SrHAp nanorods (brighter features) inside the polymeric matrix

ibility is the main problem with aliphatic polyesters and for this reason commercially available materials are prepared using a multifunctional additive as co-monomer. The addition of nano-filler cannot alter the mechanical behaviour of these nanocomposites as evidenced by the almost identical stressstrain curves that recorded breaking before the yielding point (data not shown). However, the presence of the nanoparticles in the PBSu matrix at very low concentration induces improvement of its mechanical properties. Young's modulus, increases from $408 \mathrm{MPa}$ in neat PBSu to $415 \mathrm{MPa}$ in the $\mathrm{PBSu} / \mathrm{SrHAp}$ nrds $0.5 \mathrm{wt} \%$ nanocomposite while tensile strength is also increased from and elasticity increases from $27.42 \mathrm{MPa}$ to 30.66 respectively in the nanocomposite. This is probably due to its higher molecular weight $\left(M_{\mathrm{n}} 51100 \mathrm{~g} / \mathrm{mol}\right)$ as confirmed from gel permeation chromatography and maybe because the SrHAp nanorods can act as reinforcement agents. Furthermore, the lack of extended

Table 3. Mechanical properties of neat PBSu and its nanocomposites containing different SrHAp nanorods content. Standard deviation $(\sigma)$ has been calculated using the following formula: $\sigma=\sqrt{\Sigma(x-\text { mean })^{2} / N}$.

\begin{tabular}{|l|c|c|c|}
\hline \multicolumn{1}{|c|}{ Sample } & $\begin{array}{c}\text { Tensile strength at break } \\
{[\mathbf{M P a}]}\end{array}$ & $\begin{array}{c}\text { Elongation at break } \\
{[\%]}\end{array}$ & $\begin{array}{c}\text { Young's Modulus } \\
{[\mathbf{M P a}]}\end{array}$ \\
\hline PBSu & $27.42 \pm 0.3$ & $9 \pm 0.2$ & $408 \pm 60$ \\
\hline PBSu/SrHAp nrds 0.5 wt\% & $30.66 \pm 0.3$ & $12 \pm 0.3$ & $415 \pm 50$ \\
\hline PBSu/SrHAp nrds 1.0 wt\% & $24.55 \pm 0.4$ & $8 \pm 0.4$ & $377 \pm 70$ \\
\hline PBSu/SrHAp nrds 2.5 wt\% & \multicolumn{3}{|c|}{ Brittle specimen } \\
\hline
\end{tabular}


agglomerates, which can act as points of stress concentration that promote material failure, probably contribute to this behaviour [15, 21]. For the sample $\mathrm{PBSu} / \mathrm{SrHAp}$ nrds $1.0 \mathrm{wt} \%$ both Young's modulus and elongation at break aggravated, whereas the nanocomposite $\mathrm{PBSu} / \mathrm{SrHAp}$ nrds $2.5 \mathrm{wt} \%$ was too brittle to withstand tensile testing. This deterioration of mechanical properties can be attributed to the significantly decreased molecular weight of these samples $\left(M_{\mathrm{n}}=37790 \mathrm{~g} / \mathrm{mol}\right.$ and $M_{\mathrm{n}}=21550 \mathrm{~g} / \mathrm{mol}$ respectively) compared with neat $\mathrm{PBSu}$, or it can be the result of the presence of aggregates in the polymer matrix. In general the nanoparticles act as reinforcing materials for a concentration up to $0.5 \mathrm{wt} \%$. DMA was also used to study the prepared nanocomposites and the reinforcement effect of the nanoparticles. The temperature dependence of the storage modulus $\left(E^{\prime}\right)$ and $\tan \delta$ for in situ prepared samples is presented in Figure 8. The nanocomposite PBSu/ SrHAp nrds $2.5 \mathrm{wt} \%$ could not be measured under these conditions of deformation, due to its significant brittleness. As can be seen $E^{\prime}$ gradually decreases with increasing temperature and a transition is observed between -60 and $-10^{\circ} \mathrm{C}$, which is ascribed to the glass transition temperature of PBSu (Tg) [17]. At low temperatures the storage modulus is higher compared with neat PBSu confirming the reinforcement effect of the nanorods. The increase is bigger in the case of a nanocomposite containing $0.5 \mathrm{wt} \%$ SrHAp nrds, in agreement with the results from the tensile test. This fact can be attributed to the restriction of the molecular motion of the PBSu macromolecules due to the fine dispersion of the nanofillers, which leads to increased interactions with the polymer matrix $[15,22]$. The extent of this rein-

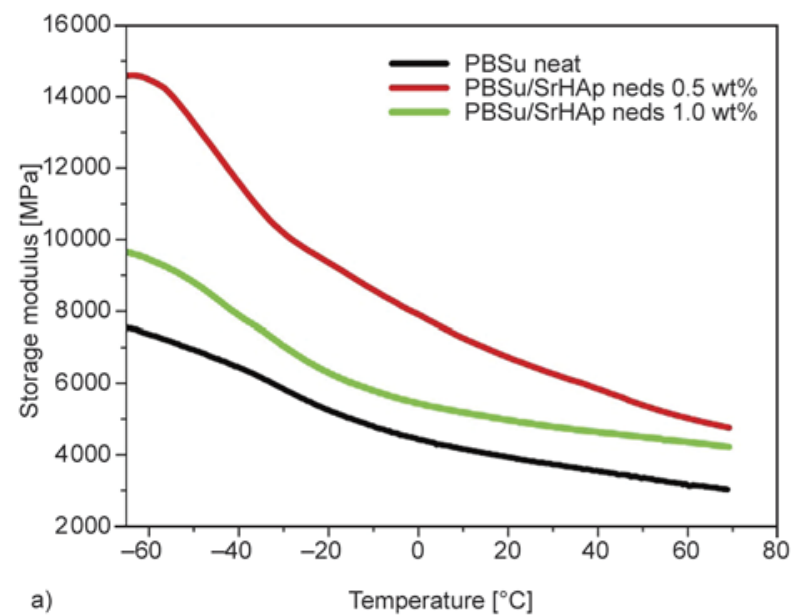

forcement is limited above the $T_{\mathrm{g}}$ due to the higher molecular motion of the PBSu macromolecular chains [17]. Thus above the $T_{\mathrm{g}}$ the storage modulus sharply decreases and all nanocomposites have almost similar storage moduli. The restricted molecular motion of the nanocomposites can also be seen from the $\tan \delta$ curve (Figure $8 b$ ). In all nanocomposites this peak has a smaller intensity, compared with neat PBSu. Furthermore, the $\tan \delta$ curve is progressively shifted to higher values by increasing the nanorod content. Such variation can be found in nanocomposites since between the polymer matrix and the nanofillers there is an interface in which, due to the interactions, the segmental mobility of macromolecules is lower compared with the matrix. However, in the present study the variation in $T_{\mathrm{g}}$ is higher $\left(6^{\circ} \mathrm{C}\right)$ than in the usual nanocomposites. This is due to the formed covalently bonds between nanorods and PBSu macromolecules, which further restricts the chain movement.

\subsection{Enzymatic hydrolysis of nanocomposites}

Specific properties are sometimes required for biomaterials. For example, tissue engineering scaffolds should have both good biocompatibility and cell adhesive properties, in addition to needed biodegradable properties [23]. Polyesters having a relatively large number of methylene groups and those having a- and b-ester bonds as PBSu with low $T_{\mathrm{m}}$ are hydrolysable by lipases [24]. As was reported in the experimental section, enzymatic hydrolysis of PBSu and its nanocomposites was studied in aqueous solutions containing a mixture of $R$. delemar and Pseudomonas Cepacia lipases, at $37^{\circ} \mathrm{C}$ and $\mathrm{pH}=$ 7.2 for 40 days. Enzymatic hydrolysis rates of the

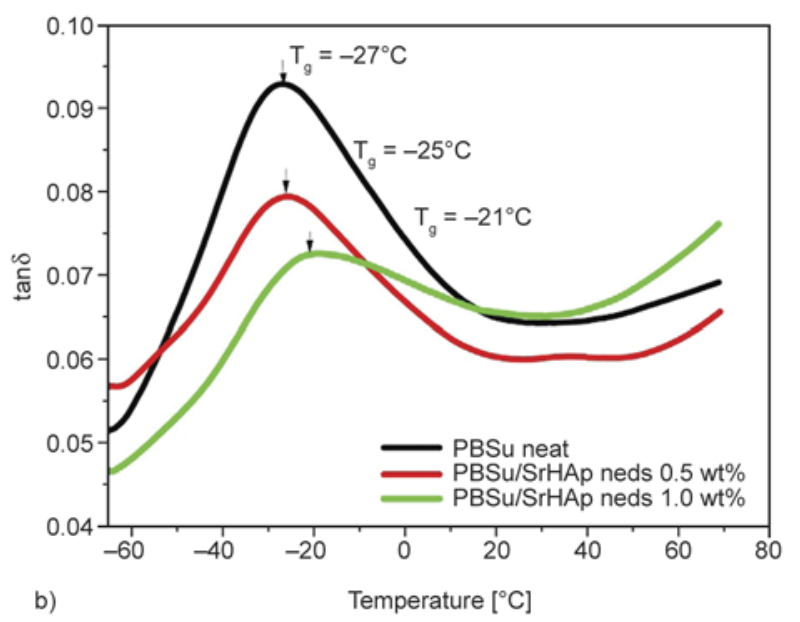

Figure 8. DMA diagrams of neat PBSu and its nanocomposites containing different SrHAp nanorods content. a) Storage modulus variation and $b$ ) $\tan \delta$ variation versus temperature. 


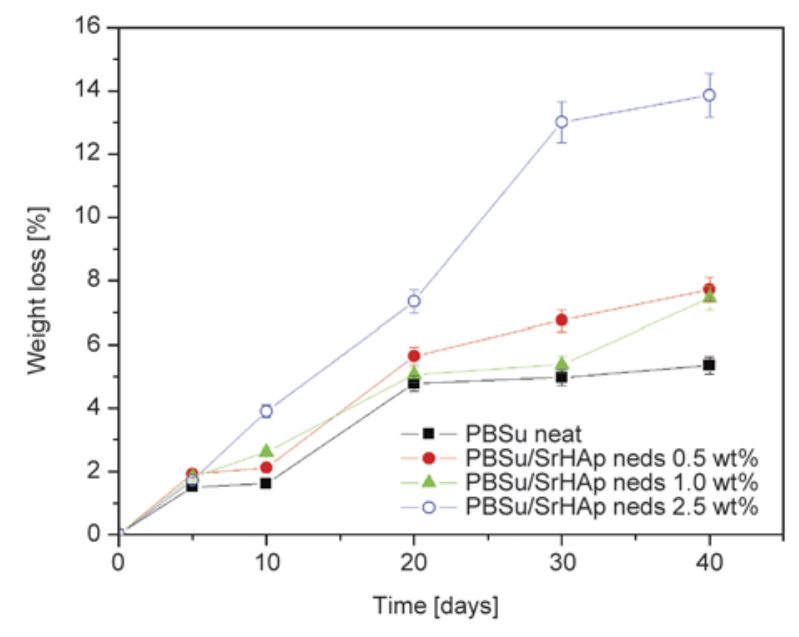

Figure 9. Mass loss graphs of neat PBSu and its nanocomposites during enzymatic hydrolysis

nanocomposites were found significantly different from those of neat PBSu. Figure 9 shows the weight loss of the specimens, expressed as a fraction of 100 (percentage) of the initial mass of the specimen as a function of time of enzymatic hydrolysis. As can be seen, pristine PBSu hydrolyzes slowly losing only about $5 \%$ of its original mass after 40 days of enzymatic treatment. The SrHAp nanorods enhance enzymatic hydrolysis and it can be seen that as the amount of nanoparticles increases, the hydrolysis rate becomes higher. PBSu/SrHAp nrds $2.5 \mathrm{wt} \%$ shows the fastest enzymatic degradation among the tested samples, losing $14 \%$ of its initial after 40 days of enzymatic hydrolysis. These results are in accordance with our previous study on PBSu/SrHAp nanorods and $\mathrm{PBSu} / \mathrm{SiO}_{2}$ nanotubes nanocomposites prepared by the melt mixing technique, where the nanocomposites reported that have higher hydrolysis rates that of neat PBSu [9]. Li et al. [25] found that $\mathrm{PLA} / \mathrm{SiO}_{2}$ nanocomposites degrade faster than neat PLA, indicating that incorporation of silica enhances biodegradation of PLA in nanocomposites too. The authors suggested that the addition of silica may lead to a facile attack of the enzyme molecule towards the ester groups of the PLA chains, because of silica's hydrophilicity. All this is in agreement with our study since the addition of SrHAp nanorods accelerates the enzymatic hydrolysis of PBSu due to the initiation of the heterogeneous hydrolysis of polyester by terminal hydroxylated edge groups [26]. However, Vasileiou et al. reported that the presence of fumed silica particles has a negative effect on the degradation level of PESu since it reduces macromolecular chain mobility. A reduced hydrolysis rate was also found in PPSeb/MMT nanocomposite, which is in agreement with some reported findings tR1 in literature [17, 27, 28].

Enzymatic degradation of polyesters can be affected by several factors, having to do with the chemical structure, the type and amount of filler, the solidstate of the degraded specimen. Hydrophilic/hydrophobic balance segments within the main chain, molecular weight, frequency of ester bonds along the macromolecular chains, as well as segmental mobility of the macromolecules can also influence the rate of biodegradation [17]. The molecular weight could be a notable factor for such differences since nanocomposites with 1.0 and $2.5 \mathrm{wt} \%$ of nanofiller have higher molecular weights than neat PBSu and this could justify the faster enzymatic hydrolysis rates observed. As the degradation rate is associated with the ability of water to wet and subsequently absorb into the nanocomposites, the hydrophilic or hydrophobic character of the nanoparticle can have a strong influence on the extent to which hydrolysis occurs. The hydrophobicity of the samples was evaluated by measuring their contact angle and water uptake Table 4. Neat PBSu has a contact angle of $76.2 \pm 0.3^{\circ}$. The addition of SrHAp nanorods seems to decrease gradually the contact angle of nanocomposites, indicating that the materials becomes more hydrophilic. This observation is in good agreement with previous work on PBSu composites, where the addition of poly(l-lactic acid) (PLLA)-grafted TTCP particles (g-TTCP) in the PBSu effectively improved its hydrophobic property [29]. PBSu/ SrHAp nanocomposites with $0.5,1$ and $2.5 \mathrm{wt} \%$ nanorods have contact angles $74 \pm 1,72.9 \pm 0.50$ and $70.3 \pm 0.2^{\circ}$ respectively. $\mathrm{PBSu} / \mathrm{SrHAp}$ nrds with $2.5 \mathrm{wt} \%$ nanorods is the most hydrophilic material due to the high hydrophilic nature of the nanofiller and thus it has the lowest contact angle. This higher hydrophilicity may explain the higher enzymatic hydrolysis rate $f$ nanocomposites compared with neat PBSu.

Table 4. Contact angle measurements of prepared nanocomposites. Standard deviation $(\sigma)$ has been calculated using the following formula:

\begin{tabular}{|l|c|}
\multicolumn{2}{|c|}{$\sigma=\sqrt{\Sigma(x-\text { mean })^{2} / N}$} \\
\hline \multicolumn{1}{|c|}{ Sample } & $\begin{array}{c}\text { Contact angle } \\
{\left[{ }^{\circ}\right]}\end{array}$ \\
\hline PBSu neat & $76.2 \pm 0.3$ \\
\hline PBSu/SrHAp nanorods $0.5 \%$ & $74.0 \pm 1.0$ \\
\hline PBSu/SrHAp nanorods $1.0 \%$ & $72.9 \pm 0.5$ \\
\hline PBSu/SrHAp nanorods $2.5 \%$ & $70.3 \pm 0.2$ \\
\hline
\end{tabular}




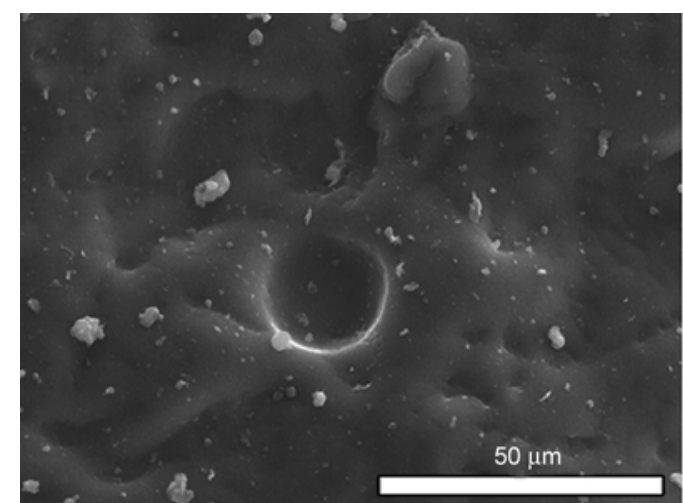

a)

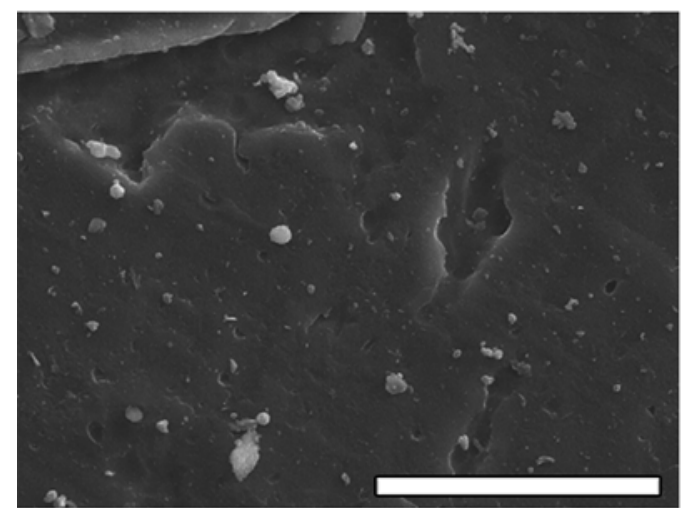

c)

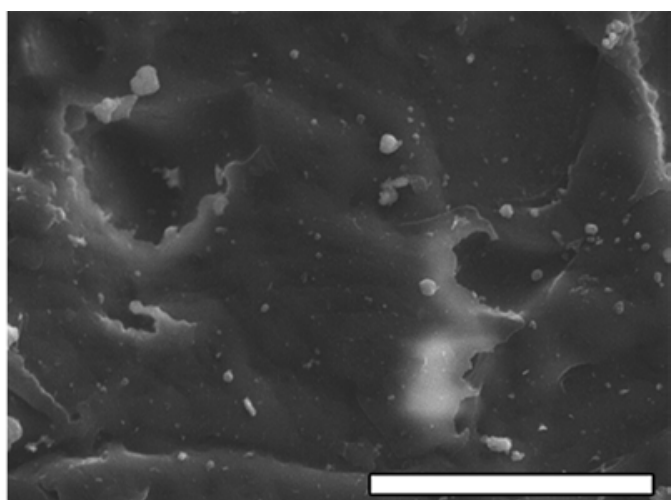

b)

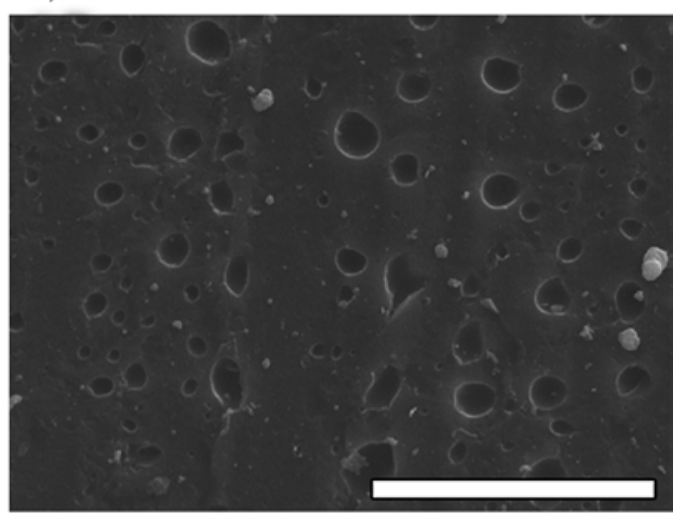

d)

Figure 10. SEM images of (a) the neat sample and the samples with b) $0.5 \mathrm{wt} \%$, (c) $1 \mathrm{wt} \%$ and (d) $2.5 \mathrm{wt} \% \mathrm{SrHAp}$ nanorods after 20 days of enzymatic hydrolysis. The scale is identical in all images.
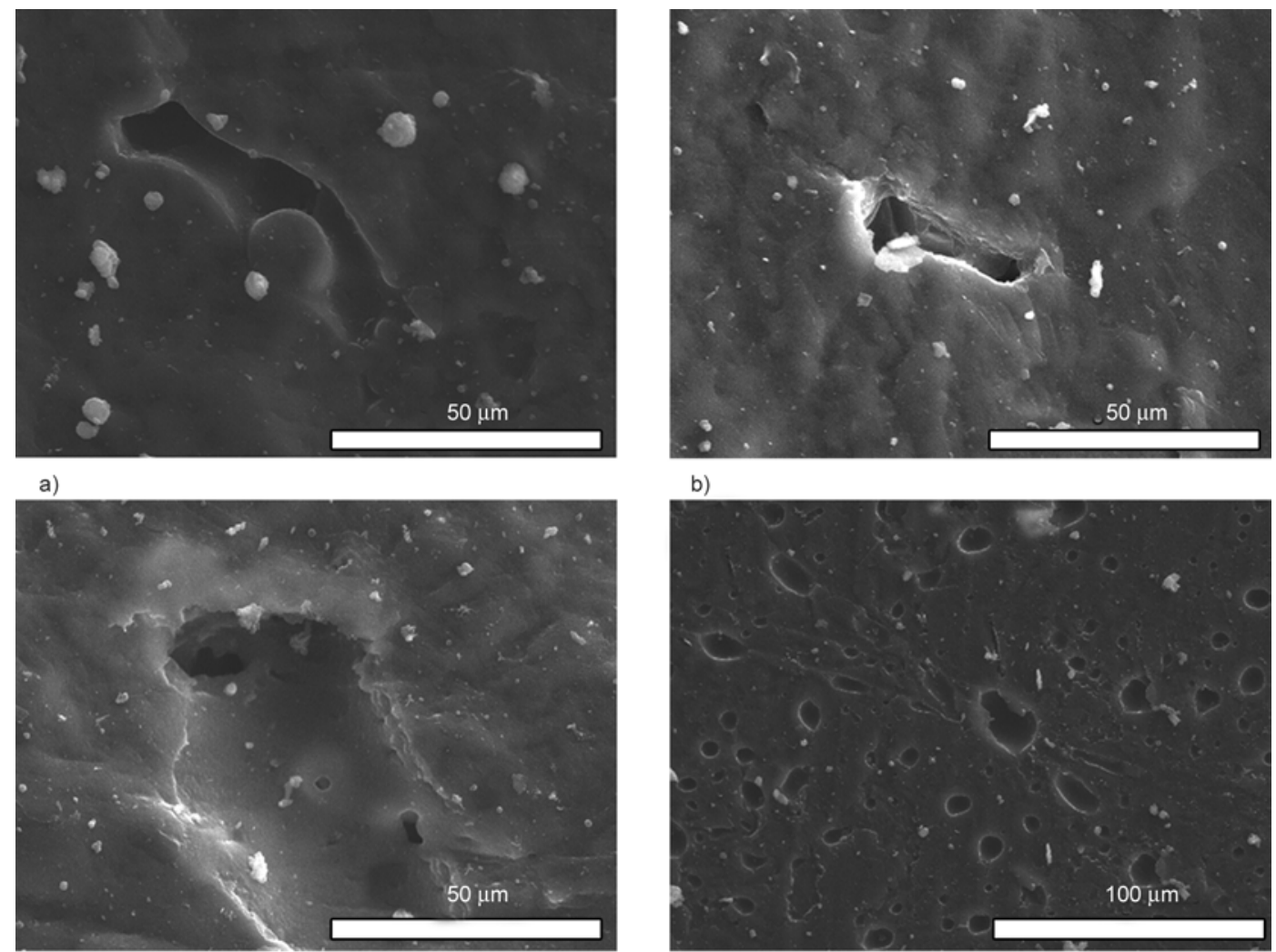

c)

Figure 11. SEM images of (a) the neat sample and the samples with (b) $0.5 \mathrm{wt} \%$, (c) $1 \mathrm{wt} \%$ and (d) $2.5 \mathrm{wt} \% \mathrm{SrHAp}$ nanorods after 40 days of enzymatic hydrolysis 
Weight loss measurements provide a general trend of the enzymatic hydrolysis, but don't reveal how this hydrolysis proceeds. For this reason, the morphology of the prepared samples before and after enzymatic hydrolysis was examined using SEM. Neat PBSu presents a less deteriorated surface with only a few cracks and holes appearing after 20 days of enzymatic hydrolysis. On the other hand, nanocomposites exhibit a much damaged surface, almost fully covered by big holes and cracks in some samples, in agreement with the mass-loss results (Figure 10). The most degraded nanocomposite is the one containing $2.5 \mathrm{wt} \%$ SrHAp nanorods, which after 40 days of hydrolysis exhibits a highly and well distributed porous-like morphology (Figure 11).

This fact can be attributed to the higher mass loss of the nanocomposites that takes place during enzymatic hydrolysis. In our previous work using higher amounts of nanorods ( 5 and $20 \mathrm{wt} \%$ ) and due to their low adhesion with the PBSu matrix, a 'gap' between PBSu and inorganic nanoparticles was formed and lipases could diffuse inside the polymer matrix forming a porous structure. The formation of such a gap and porous structure is known to accelerate the enzymatic degradation of biodegradable polyesters [30]. However, in the present study such a 'gap' was not found from SEM measurements and the higher rate of enzymatic hydrolysis of nanocomposites should be attributed to the higher hydrophylicity of the added nanorods.

\subsection{In vitro bioactivity test}

A bioactive material is defined as a material that elicits a specific biological response at the interface of the material which results in the formation of a bond between the tissue and the material. Kokubo et al. [31] suggested that for the bioactive glasses forming surface silanols upon hydration, a silica hydrogel layer is produced by exchange of ions in the $\mathrm{SBF}$, and that this layer acts as a nucleation agent for apatite crystal formation. Once the apatite crystals are formed, they grow by consuming calcium and phosphate ions from the SBF [31]. Given the similarities in composition between bioglass and polymeric systems, many authors have suggested that the formation of hydroxyapatite on the polymer surface may follow a similar mechanism [32, 33]. Figure 12 shows the morphology of PBSu after soaking in SBF for 14 days. Nucleation and growth have occurred on the exposed surfaces, resulting in clus- ters of mineral precipitates. A similar behaviour was found also in PBSu/SrHAp nrds nanocomposites (Figure 13). Most of the PBSu and PBSu/SrHAp nrds nanocomposites' surfaces were covered by a needle-like apatite layer as shown in Figures 12 and 13. As can be seen from the SEM micrographs apatite layers are further thickened and it was found that a cauliflower-like apatite cluster formed on the surface of these samples. This finding is in agreement with a previous study on plasma-treated PBSu scaffolds [7]. The results obtained by SEM are confirmed by EDX analysis (Figure 12b and Figure 13b) and a significant variation in the intensity of $\mathrm{Si}, \mathrm{P}$, and $\mathrm{Ca}$ can be observed in PBSu nanocomposites with different content of SrHAp nanorods. After soaking in SBF, the concentration of $\mathrm{Ca}$ and $\mathrm{P}$ increase, accompanied by the increase of SrHAp naorods in the PBSu matrix for a concentration of the nanofiller up to $1.0 \mathrm{wt} \%$. These results confirm to some extent our initial expectations derived from the literature, that when nanophase (compared to conventional) particles are incorporated into the polymer composite they promote mineral deposition onto the surfaces [34-36].

To characterize the inorganic phase formed onto the surfaces of PBSu and its nanocomposites, XRD analysis was performed on neat $\mathrm{PBSu}$ and its nanocomposites after soaking in SBF for 14 days (Figure 14). PBSu is a typical semicrystalline polymer with two characteristic peaks at $2 \theta 19.8$ and $22.6^{\circ}$ while the features of interest consists of a broad contour between $2 \theta=27-37^{\circ}$ resulting from the 002 reflection of hydroxyapatite. New peaks are observed at $2 \theta 32,436,45$ and $46^{\circ}$ after immersion in SBF which confirm that partially crystallized apatite layer formed on the surfaces of PBSu film as well as on its nanocomposites [33, 37, 38].

\subsection{Cell studies}

The metabolic activity of MG-63 cells cultivated for $48 \mathrm{~h}$ is illustrated in Figure 15. In all cases, except for the scaffold with $0.5 \mathrm{wt} \%$ of $\mathrm{Sr}$, the metabolic activity of MG63 cells was significantly $(p<0.05)$ reduced compared to the reference. However, the metabolic activity of all materials was higher than $50 \%$, which is considered as the limit for biocompatibility [39].

These results were further confirmed by SEM after $48 \mathrm{~h}$ of cultivation (Figure 16). Although it is hard to detect the dense MG-63 cell monolayer, in all 


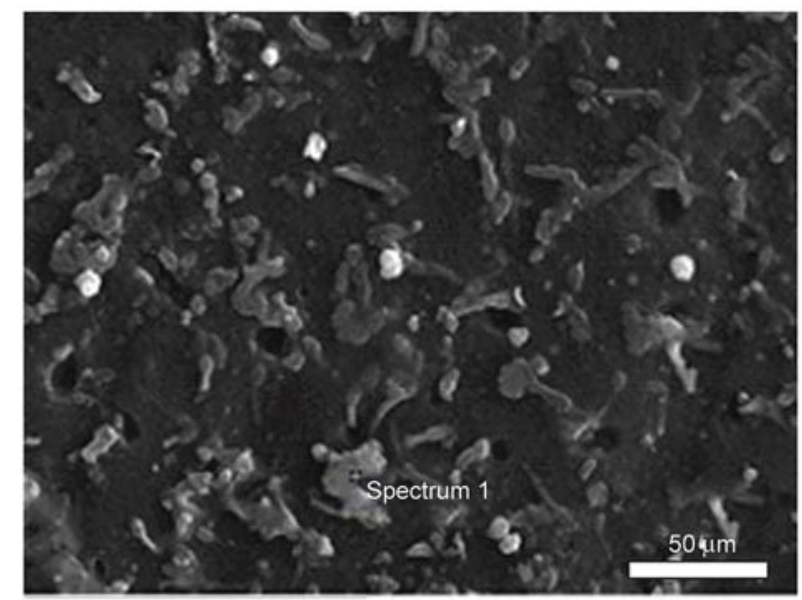

a)

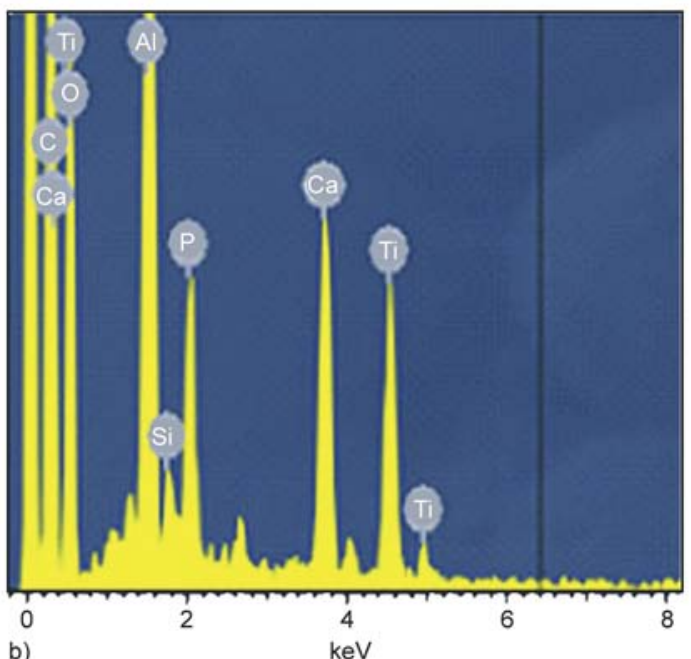

keV

Figure 12. a) SEM micrographs of neat PBSu after soaking in SBF for 14 days and b) EDX analysis of PBSu surface indicating the formation of hydroxyapatite

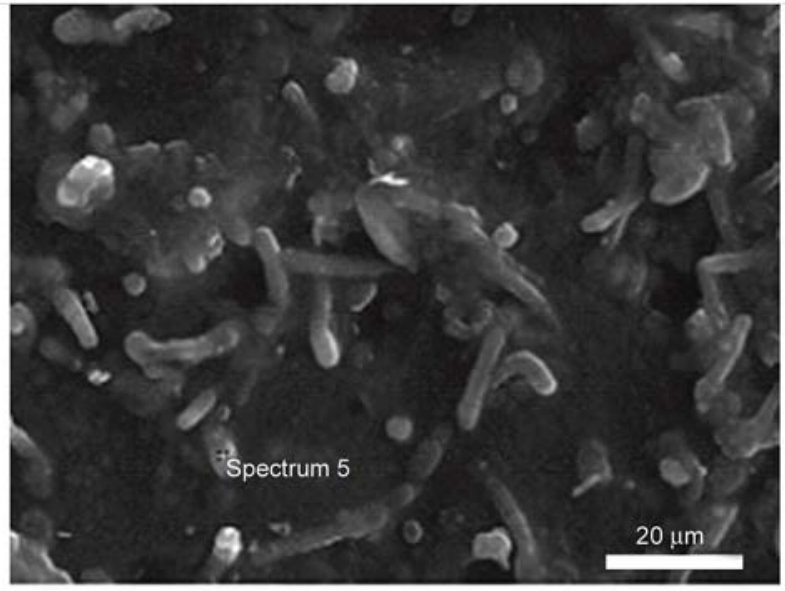

a)

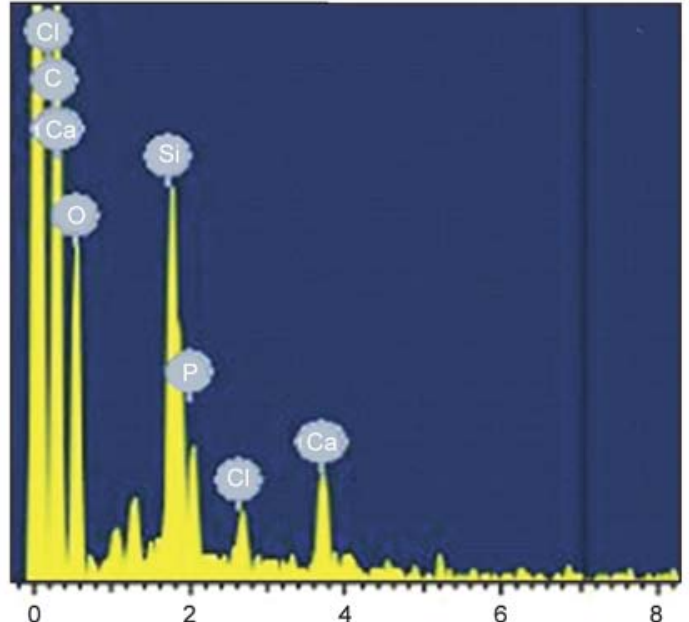

b)

keV

Figure 13. a) SEM micrographs of PBSu/SrHAp nrds containing $0.5 \mathrm{wt} \%$ nanorods after soaking in SBF for 14 days and b) EDX analysis of nanocomposites surface indicating the formation of hydroxyapatite

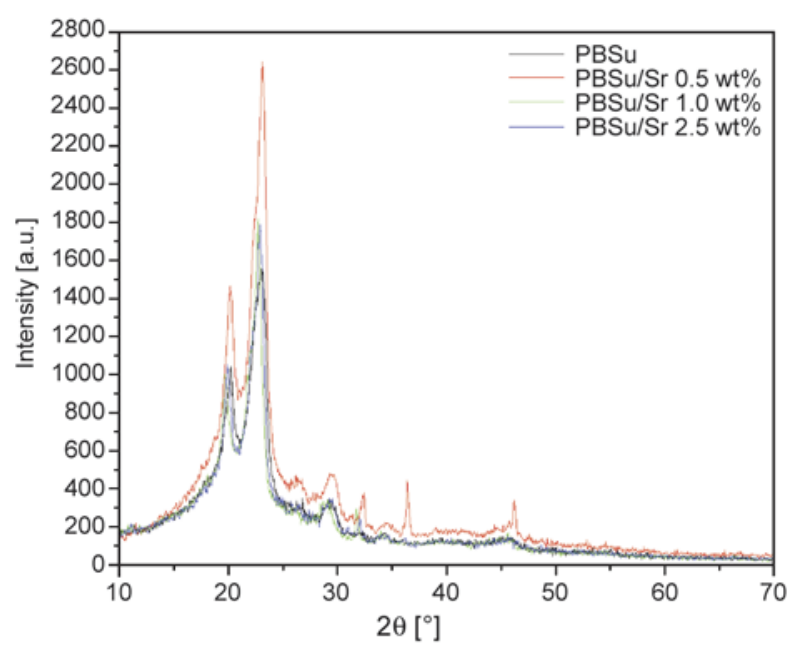

Figure 14. XRD patterns of neat PBSu and its nanocomposites after soaking in SBF for 14 days

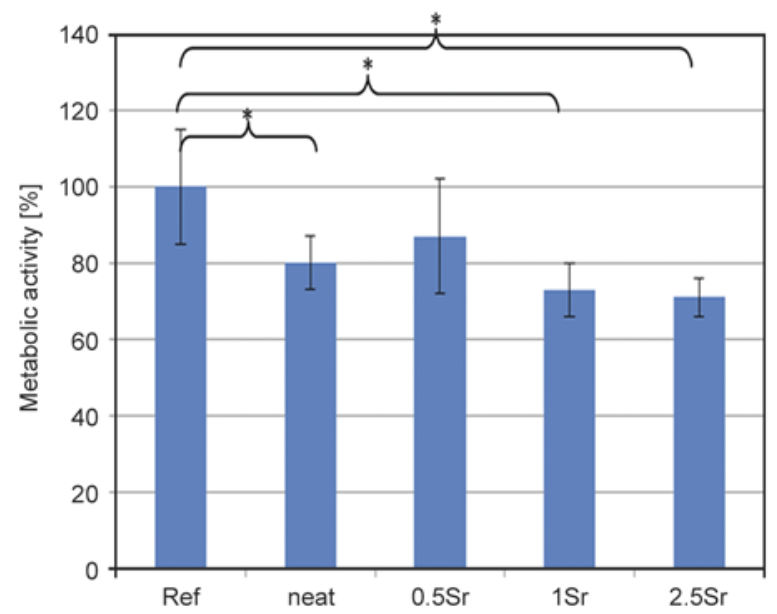

Figure 15. Metabolic activity of MG-63 cells cultivated for $48 \mathrm{~h}$ on plane composite samples 


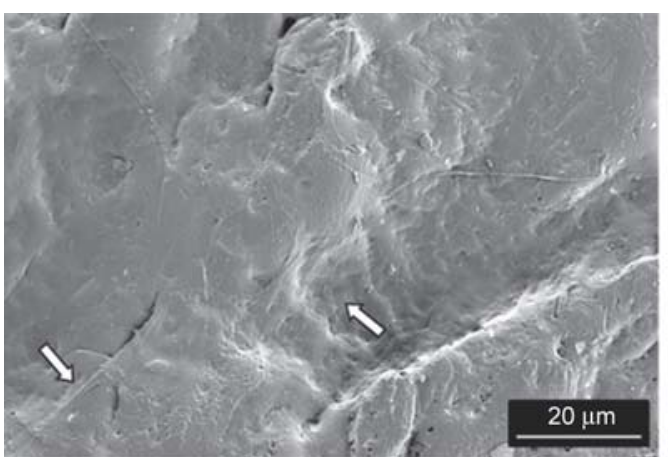

a)

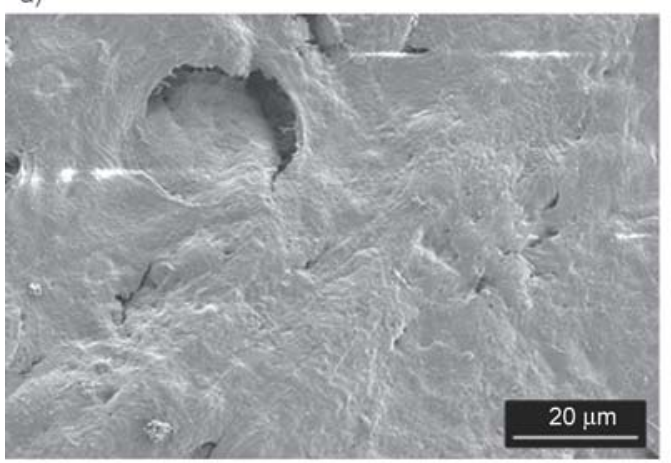

c)

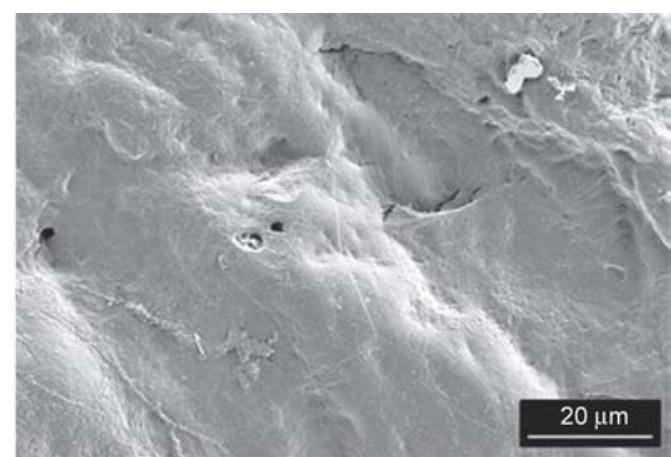

b)

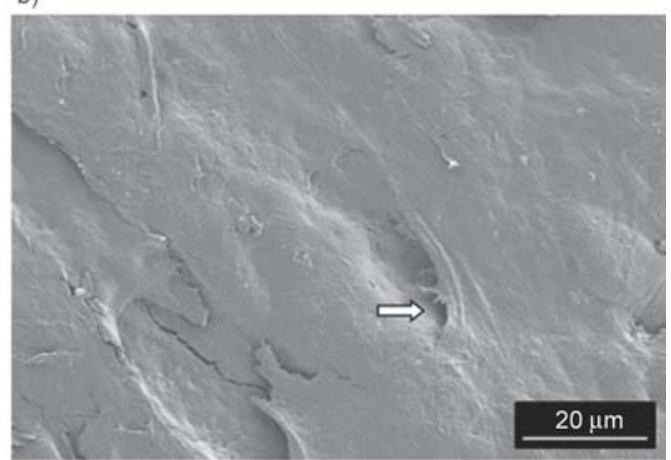

d)

Figure 16. SEM images of (a) the neat sample and the samples with (b) $0.5 \mathrm{wt} \%$, (c) $1 \mathrm{wt} \% \mathrm{Sr}$ and (d) $2.5 \mathrm{wt} \%$ nanorods after incubation with MG63 for 48 hours

cases the cells have attached and spread on the entire surface of the samples. As the expressed morphology of the MG-63 cells on the different surfaces are very flat, no obvious difference in cell behaviour could be analysed applying the different scaffold compositions.

In summary, although a significant reduction of metabolic activity was measured in MG-63 after $48 \mathrm{~h}$ of incubation on the test samples compared to the reference, no difference was shown in the cell morphology study. It can therefore be concluded that the tested materials are biocompatible in vitro and further in vitro and in vivo studies have to be carried out to confirm the osteogenic potential of the developed materials in the context of bone regeneration.

\section{Conclusions}

SrHAp nrds have been hydrothermally synthesized. Afterwards, PBSu nanocomposites containing SrHAp nrds at different concentrations have been in situ prepared and studied as potential materials for tissue engineering applications. GPC analysis verified that macromolecules with satisfying $M_{\mathrm{w}}$ and $M_{\mathrm{n}}$ values were obtained while XPS spectra indicated the presence of some interactions between the inor- ganic filler and the PBSu matrix. The nanocomposite containing $0.5 \mathrm{wt} \%$ SrHAp nrds, improved the mechanical properties but further increase of the nanofiller content up to $2.5 \mathrm{wt} \%$ caused a deterioration of the mechanical properties as a result of the bad dispersion in the PBSu matrix. It was also found that the nanofiller enhanced enzymatic hydrolysis probably due to the initiation of heterogeneous hydrolysis of the polyester by terminal hydroxylated edge groups. SrHAp nrds can transform the bioinert PBSu surface into a bioactive one, promoting hydroxyapatite formation in SBF solution. In addition to the apatite formation enhancement, SrHAp nrds can induce osteoblast compatibility. Nanocomposites have more hydrophilic surfaces compared to pristine $\mathrm{PBSu}$, thereby causing more osteoblasts to adhere and proliferate on them.

\section{Acknowledgements}

The authors wish to acknowledge co-funding of this research by IKY (Greece) and DAAD (Germany), Action 'IKYDA 2012' and the financial support from the European Union under the Seventh Framework Program (Integrated Infrastructure Initiative No. 262348 European Soft Matter Infrastructure, ESMI). 


\section{References}

[1] Ouchi T., Ohya Y.: Design of lactide copolymers as biomaterials. Journal of Polymer Science Part A: Polymer Chemistry, 42, 453-462 (2003).

DOI: 10.1002/pola.10848

[2] Puskas J. E., Chen Y.: Biomedical application of commercial polymers and novel polyisobutylene-based thermoplastic elastomers for soft tissue replacement. Biomacromolecules, 5, 1141-1154 (2004).

DOI: $10.1021 / \mathrm{bm} 034513 \mathrm{k}$

[3] Sabir M. I., Xu X., Li L.: A review on biodegradable polymeric materials for bone tissue engineering applications. Journal of Materials Science, 44, 5713-5724 (2009).

DOI: $10.1007 / \mathrm{s} 10853-009-3770-7$

[4] Li H., Chang J., Cao A., Wang J.: In vitro evaluation of biodegradable poly(butylene succinate) as a novel biomaterial. Macromolecular Bioscience, 5, 433-440 (2005).

DOI: 10.1002/mabi.200400183

[5] Vroman I., Tighzert L.: Biodegradable polymers. Materials, 2, 307-344 (2009).

DOI: $10.3390 / \mathrm{ma} 2020307$

[6] Costa-Pinto A. R., Correlo V. M., Sol P. C., Bhattacharya M., Srouji S., Livne E., Reis R. L., Neves N. M.: Chitosan-poly(butylene succinate) scaffolds and human bone marrow stromal cells induce bone repair in a mouse calvaria model. Journal of Tissue Engineering and Regenerative Medicine, 6, 21-28 (2012).

DOI: $10.1002 /$ term.391

[7] Wang H., Ji J., Zhang W., Zhang Y., Jiang J., Wu Z., Pu S., Chu P. K:. Biocompatibility and bioactivity of plasma-treated biodegradable poly(butylene succinate). Acta Biomaterialia, 5, 279-287 (2009).

DOI: $10.1016 /$ j.actbio.2008.07.017

[8] Christenson E. M., Anseth K. S., van den Beucken J. J. J. P., Chan C. K., Ercan B., Jansen J. A., Laurencin C. T., Li W-J., Murugan R., Nair L. S., Ramakrishna S., Tuan R. S., Webster T. J., Mikos A. G.: Nanobiomaterial applications in orthopedics. Journal of Orthopaedic Research, 25, 11-22 (2007).

DOI: $10.1002 /$ jor.20305

[9] Grigoriadou I., Nianias N., Hoppe A., Terzopoulou Z., Bikiaris D., Will J., Hum J., Roether J. A., Detsch R., Boccaccini A. R.: Evaluation of silica-nanotubes and strontium hydroxyapatite nanorods as appropriate nanoadditives for poly(butylene succinate) biodegradable polyester for biomedical applications. Composites Part B: Engineering, 60, 49-59 (2014).

DOI: 10.1016/j.compositesb.2013.12.015

[10] Zhang C., Li C., Huang S., Hou Z., Cheng Z., Yang P., Peng C., Lin J.: Self-activated luminescent and mesoporous strontium hydroxyapatite nanorods for drug delivery. Biomaterials, 31, 3374-3383 (2010).

DOI: $10.1016 /$ j.biomaterials.2010.01.044
[11] Ahn B. D., Kim S. H., Kim Y. H., Yang J. S.: Synthesis and characterization of the biodegradable copolymers from succinic acid and adipic acid with 1,4-butanediol. Journal of Applied Polymer Science, 82, 2808-2826 (2001). DOI: $10.1002 / a p p .2135$

[12] Bikiaris D., Karavelidis V., Karayannidis G.: A new approach to prepare poly(ethylene terephthalate)/silica nanocomposites with increased molecular weight and fully adjustable branching or crosslinking by SSP. Macromolecular Rapid Communications, 27, 11991205 (2006). DOI: $10.1002 /$ marc. 200600268

[13] Vassiliou A. A., Chrissafis K., Bikiaris D. N.: In situ prepared $\mathrm{PBSu} / \mathrm{SiO}_{2}$ nanocomposites. Study of thermal degradation mechanism. Thermochimica Acta, 495, 120-128 (2009).

DOI: $10.1016 /$ j.tca.2009.06.010

[14] Han S-I., Lim J. S., Kim D. K., Kim M. N., Im S. S.: In situ polymerized poly(butylene succinate)/silica nanocomposites: Physical properties and biodegradation. Polymer Degradation and Stability, 93, 889-895 (2008). DOI: 10.1016/j.polymdegradstab.2008.02.007

[15] Vassiliou A. A., Bikiaris D., Mabrouk K., Kontopoulou M.: Effect of evolved interactions in poly(butylene succinate)/fumed silica biodegradable in situ prepared nanocomposites on molecular weight, material properties, and biodegradability. Journal of Applied Polymer Science, 119, 2010-2024 (2010). DOI: $10.1002 / a p p .32887$

[16] Zhang W., Nefedov A., Naboka M., Cao L., Wöll C.: Molecular orientation of terephthalic acid assembly on epitaxial graphene: NEXAFS and XPS study. Physical Chemistry Chemical Physics, 14, 10125-10131 (2012). DOI: $10.1039 / \mathrm{C} 2 \mathrm{CP} 23748 \mathrm{~B}$

[17] Bikiaris D. N., Nianias N. P., Karagiannidou E. G., Docoslis A.: Effect of different nanoparticles on the properties and enzymatic hydrolysis mechanism of aliphatic polyesters. Polymer Degradation and Stability, 97, 2077-2089 (2012). DOI: $10.1016 /$ j.polymdegradstab.2011.10.024

[18] Wang H., Ji J., Zhang W., Wang W., Zhang Y., Wu Z., Zhang Y., Chu P. K.: Rat calvaria osteoblast behavior and antibacterial properties of $\mathrm{O}_{2}$ and $\mathrm{N}_{2}$ plasmaimplanted biodegradable poly(butylene succinate). Acta Biomaterialia, 6, 154-159 (2010). DOI: $10.1016 /$ j.actbio.2009.07.026

[19] Xia W., Lindahl C., Persson C., Thomsen P., Lausmaa J., Engqvist H.: Changes of surface composition and morphology after incorporation of ions into biomimetic apatite coating. Journal of Biomaterials and Nanobiotechnology, 1, 7-16 (2010).

DOI: $10.4236 /$ jbnb.2010.11002 
[20] Fan R. R., Zhou L. X., Li D. X., Zhang D. M., Wu M., Guo G.: Preparation and characterization of composites based on poly(butylene succinate) and poly(lactic acid) grafted tetracalcium phosphate. Journal of Macromolecular Science Part B: Physics, 53, 296-308 (2013).

DOI: $10.1080 / 00222348.2013 .810104$

[21] Albertsson A. C., Verma I. K.: Aliphatic polyesters: Synthesis, properties and applications. Advances in Polymer Science, 157, 1-40 (2002). DOI: $10.1007 / 3-540-45734-8 \quad 1$

[22] Kontou E., Niaounakis M.: Thermo-mechanical properties of LLDPE/SiO ${ }_{2}$ nanocomposites. Polymer, 47, 1267-1280 (2006).

DOI: 10.1016/j.polymer.2005.12.039

[23] Liu X., Ma P. X.: Polymeric scaffolds for bone tissue engineering. Annals of Biomedical Engineering, 32, 477-486 (2004).

DOI: 10.1023/B:ABME.0000017544.36001.8e

[24] Herzog K., Müller R-J., Deckwer W-D.: Mechanism and kinetics of the enzymatic hydrolysis of polyester nanoparticles by lipases. Polymer Degradation and Stability, 91, 2486-2498 (2006).

DOI: $10.1016 /$ j.polymdegradstab.2006.03.005

[25] Li Y., Han C., Bian J., Han L., Dong L., Gao G.: Rheology and biodegradation of polylactide/silica nanocomposites. Polymer Composites, 33, 1719-1727 (2012). DOI: $10.1002 /$ pc.22306

[26] Fukushima K., Tabuani D., Camino G.: Nanocomposites of PLA and PCL based on montmorillonite and sepiolite. Materials Science and Engineering: C, 29, 1433-1441 (2009).

DOI: $10.1016 / \mathrm{j} \cdot \mathrm{msec} .2008 .11 .005$

[27] Lee S-R., Park H-M., Lim H., Kang T., Li X., Cho WJ., Ha C-S.: Microstructure, tensile properties, and biodegradability of aliphatic polyester/clay nanocomposites. Polymer, 43, 2495-2500 (2002).

DOI: $10.1016 / \mathrm{S} 0032-3861(02) 00012-5$

[28] Nerantzaki M., Papageorgiou G. Z., Bikiaris D. N.: Effect of nanofiller's type on the thermal properties and enzymatic degradation of poly( $\varepsilon$-caprolactone). Polymer Degradation and Stability, 108, 257-268 (2014).

DOI: $10.1016 /$ j.polymdegradstab.2014.03.018

[29] Fan R. R., Zhou L. X., Song W., Li D. X., Zhang D. M., Ye R., Zheng Y., Guo G.: Preparation and properties of g-TTCP/PBS nanocomposites and its in vitro biocompatibility assay. International Journal of Biological Macromolecules, 59, 227-234 (2013).

DOI: $10.1016 /$ j.ijbiomac.2013.04.051
[30] Tsuji H., Kawashima Y., Takikawa H., Tanaka S.: Poly(L-lactide)/nano-structured carbon composites: Conductivity, thermal properties, crystallization, and biodegradation. Polymer, 48, 4213-4225 (2007).

DOI: $10.1016 /$ j.polymer.2007.05.040

[31] Kokubo T., Kushitani H., Sakka S., Kitsugi T., Yamamuro T.: Solutions able to reproduce in vivo surfacestructure changes in bioactive glass-ceramic $\mathrm{A}-\mathrm{W}^{3}$. Journal of Biomedical Materials Research, 24, 721734 (1990). DOI: $10.1002 / \mathrm{jbm} .820240607$

[32] Chouzouri G., Xanthos M.: In vitro bioactivity and degradation of polycaprolactone composites containing silicate fillers. Acta Biomaterialia, 3, 745-756 (2007). DOI: $10.1016 / \mathrm{j}$.actbio.2007.01.005

[33] Hong Z., Reis R. L., Mano J. F.: Preparation and in vitro characterization of novel bioactive glass ceramic nanoparticles. Journal of Biomedical Materials Research Part A, 88, 304-313 (2009).

DOI: $10.1002 / \mathrm{jbm} . \mathrm{a} .31848$

[34] Wei G., Ma P. X.: Structure and properties of nanohydroxyapatite/polymer composite scaffolds for bone tissue engineering. Biomaterials, 25, 4749-4757 (2004). DOI: $10.1016 /$ j.biomaterials.2003.12.005

[35] Webster T. J., Smith T. A.: Increased osteoblast function on PLGA composites containing nanophase titania. Journal of Biomedical Materials Research Part A, 74, 677-686 (2005).

DOI: $10.1002 / \mathrm{jbm} . \mathrm{a} .30358$

[36] Peter M., Binulal N. S., Soumya S., Nair S. V., Furuike T., Tamura H., Jayakumar R.: Nanocomposite scaffolds of bioactive glass ceramic nanoparticles disseminated chitosan matrix for tissue engineering applications. Carbohydrate Polymers, 79, 284-289 (2010). DOI: $10.1016 /$ j.carbpol.2009.08.001

[37] Chavan P. N., Bahir M. M., Mene R. U., Mahabole M. P., Khairnar R. S.: Study of nanobiomaterial hydroxyapatite in simulated body fluid: Formation and growth of apatite. Materials Science and Engineering: B, 168, 224-230 (2010).

DOI: $10.1016 /$ j.mseb.2009.11.012

[38] Gadaleta S. J., Paschalis E. P., Betts F., Mendelsohn R., Boskey A. L.: Fourier transform infrared spectroscopy of the solution-mediated conversion of amorphous calcium phosphate to hydroxyapatite: New correlations between X-ray diffraction and infrared data. Calcified Tissue International, 58, 9-16 (1996).

DOI: $10.1007 / \mathrm{BF} 02509540$

[39] Bruinink A., Luginbuehl R.: Evaluation of biocompatibility using in vitro methods: Interpretation and limitations. Advances in Biochemical Engineering/Biotechnology, 126, 117-152 (2012).

DOI: $10.1007 / 10 \quad 2011 \quad 111$ 